\title{
Determining EDS and EELS partial cross-sections from multiple calibration standards to accurately quantify bi-metallic nanoparticles using STEM.
}

\author{
Aakash Varambhia ${ }^{1}$, Lewys Jones ${ }^{2,3}$, Andrew London ${ }^{1}$, Dogan Ozkaya ${ }^{4}$, \\ Peter D Nellist ${ }^{1}$, Sergio Lozano-Perez*1
}

1. Department of Materials, University of Oxford, Parks Road, OX1 3PH, UK

2. School of Physics, Trinity College Dublin, Dublin 2, Ireland

3. Advanced Microscopy Laboratory, Centre for Research on Adaptive Nanostructures and Nanodevices (CRANN), Dublin 2, Ireland

4. Johnson Matthey Technology Centre, Sonning Common, Reading, RG4 9NH, UK

Corresponding author email: sergio.lozano-perez@materials.ox.ac.uk

\begin{abstract}
.
Spectroscopic signals such as EDS and EELS provide an effective way of characterising multielement samples such as Pt-Co nanoparticles in STEM. The advantage of spectroscopy over imaging is the ability to decouple composition and mass-thickness effects for thin samples, into the number of various types of atoms in a sample. This is currently not possible for multi element samples using conventional ADF quantification techniques alone. With recent developments in microscope hardware and software, it is now possible to acquire the ADF, EDS and EELS signals simultaneously and at high speed. However, the methods of quantifying the signals emitted from the sample vary greatly. Most approaches use pure-element standards in the form of needles, nanoparticles and wedges to quantify the spectroscopic signal into either partial scattering cross-sections, zeta-factors or k-factors. But self-consistency between the different methods has not been verified and the units of the quantification are not standardised. We present a robust approach for measuring and combining ADF, EDS and EELS signals using needle and nanoparticle standards in units of the partial scattering crosssection. The partial scattering cross-section allows an easy interpretation of the signals emitted from the sample and enables accurate atom-counting of the sample.

- Using known element needle and nanoparticle standards to quantify spectroscopic signals consistently.

- Using simultaneously acquired signals to independently quantify a sample in the electron microscope.
\end{abstract}

Kew Words: Quantitative Spectroscopy, EDS, EELS, STEM, ADF

\section{Introduction.}

To quantify industrially relevant materials such as bimetallic Pt-Co catalyst nanoparticles, a thorough characterisation of their size, shape and composition is required. Catalyst systems such as Pt-Co are of particular interest in the fuel cell industry due to their enhanced activity compared to pure element catalyst counterparts (Koh and Strasser, 2007; Liu et al., 2013; Xin et al., 2012).

Currently, quantitative size and shape information can be routinely obtained from pure element nanoparticles using quantitative Annular Dark Field (ADF) Scanning Transmission Electron Microscopy (STEM) (Aarons et al., 2017). Composition information is also visible in ADF imaging (Liu and Cowley, 1990), but for bimetallic nanoparticle systems, the ADF signal alone is not sufficient in decoupling the intensity variations caused by composition and thickness changes. To decouple these effects, a spectroscopic approach such as Energy Dispersive X-ray Spectroscopy 
(EDS) or Electron Energy Loss Spectroscopy (EELS) is required. In both spectroscopic approaches, the intensity obtained can be interpreted by observation of the strength of the signals at characteristic excitation energies for a given element. In principle, measured intensities from ADF, EDS and EELS can all be interpreted in terms of an appropriate partial scattering cross-section (E et al., 2013; Egerton, 2008; Macarthur et al., 2015; Martinez et al., 2015). Here we present a method for experimentally determining EDS and EELS cross-sections, and, more importantly, verify their accuracy by demonstrating consistency of counting atoms in nanoparticles across measurements made using ADF, EDS and EELS.

The cross-section we measure is described as partial because it depends on factors such as the collection-angle, collection-efficiency, and the size and shape of the detectors in question. Additionally, due to detection reliability and counting statistics, sometimes not all ionization edges of the sample are incorporated in the measurement. Since most of these factors are microscope dependent, the partial cross-section is an excellent parameter to benchmark detector performance between microscopes (Macarthur et al., 2015).

Multiple attempts have been made at performing quantification using standards in the past (Cliff and Lorimer, 1975; Watanabe et al., 1996). However, for small nanoparticles, detector and software limitations have made this quantification difficult and time consuming. With the recent increase in EDS collection-efficiency using new silicon drift detectors (Phillips et al., 2014) and improvements to the Gatan Image Filter (GIF) (Gubbens et al., 2010) it is now possible, with sufficient speed and counting statistics, to obtain both EDS and EELS signals simultaneously alongside the ADF signal (Kothleitner et al., 2014). Our aim is, that by collecting signals simultaneously from ADF and spectroscopic detectors, thickness and composition effects can be decoupled completely for the highest precision quantification of nanoparticles composition and structure.

The measurement of ADF cross-sections has become well established (Aarons et al., 2017; E et al., 2013; Jones et al., 2014) and relies on careful detector calibration (Jones et al., 2017) and conversion to atom counts through simulation matching techniques (Aarons et al., 2017), statistical decomposition (De Backer et al., 2013; Van Aert et al., 2011) or a hybrid of the two (De wael et al., 2017).

In contrast, spectroscopic quantification has made use of the following methods: k-factor(EDS)(Cliff and Lorimer, 1975), $\zeta$ (zeta)-factor(EDS)(Watanabe and Williams, 2006) or a partial scattering crosssection (EELS and EDS) (Craven et al., 2016; Macarthur et al., 2015). Within these approaches the most popular quantification techniques are the k-factor approach for EDS and the Hartree Slater cross-section approach for EELS. These techniques owe their popularity to out-of-the-box incorporation within commercially available software. As these techniques rely on theoretically calculated models, they may contain systematic errors of up to $10 \%$ in some cases (Watanabe et al., 2003).

The alternative but more tedious approach is to measure the k-factor and the scattering cross-section experimentally. Here difficulties emerge due to sample preparation, as the k-factor approach requires a thin well-characterised binary sample and the cross-section approach requires a pure element sample thin enough for minimal multiple scattering. In order to tackle the binary sample problem, (Watanabe et al., 1996) proposed the pure element zeta factor method to quantify the EDS signal. Building upon this, (Kothleitner et al., 2014) proposed a method to quantify the EDS and EELS signal using a pure element needle standard. Here the thickness of the needle can be directly measured by physically tilting the needle by $90^{\circ}$. Here the EDS signal was quantified using a zeta factor and the EELS signal was quantified using a partial scattering cross-section. The zeta-factor and the partial cross-section were then linked by a conversion factor for multivariate elemental analysis. However, no attempt was made at representing the zeta-factor as a scattering cross-section. 
To standardise the ADF, EDS and EELS units, (Macarthur et al., 2015) proposed a wedge sample approach to convert the zeta factor into a EDS partial scattering cross-section. But determining the zthickness for a wedge sample is difficult, and may lead to systematic errors in quantification (Macarthur et al., 2015). While a standard-less simulation matching based technique has been demonstrated for EDS signals, it is difficult to implement (Chen et al., 2016). In this technique, all parameters of the microscope must be characterised meticulously for a good agreement with theory, but even here, some discrepancies remain (Chen et al., 2016; Findlay et al., 2017). Because of the tedious nature of this technique, standard-based techniques remain popular as the microscope calibrations required are fewer and easier.

Recently, (Zanaga et al., 2016) proposed a method to quantify the EDS signal in zeta-factors using tomography and nanoparticles as a known standard. But no verification was made to check the accuracy of the quantification against established methods using wedge or needle standards. In the same year, (Craven et al., 2016) proposed a method of determining EELS partial cross-sections using experimentally measured electron mean free paths, but no comparison to EDS cross-sections was made.

In this paper we aim to validate and verify the accuracy of measured partial scattering cross-sections using both needle and nanoparticle standards. Not only does this increase the accuracy of the quantification, but also allows for quantification of samples where the known element standard is difficult to synthesise. For example, to quantify the Co concentration in Pt-Co nanoparticles a pure Co standard is required. However, synthesising a pure Co nanoparticle standard is difficult due to Co readily oxidising when exposed to air. Alternatively, ion-milling a bulk material Co needle is much easier and a single atom Co partial cross-section can be measured. Thus, the accuracy between needle and nanoparticle known element standards needs to be verified.

Additionally, representing the ADF, EDS and EELS signals as partial scattering cross-sections simplifies quantification and allows for benchmarking of different microscopes. Here we benchmark the measured EDS cross-section on two JEOL ARM 200CF microscopes, one with a single EDS detector and the other with a double EDS detector.

We also show how the EDS and EELS signals can be linked through a constant when the spectroscopic signal is represented in partial scattering cross-section. Then with the measured partial cross-sections, the concentration within a sample can be quantified using independent, but simultaneously acquired, signals. As an example, an on-axis nanoparticle was atom counted using $\mathrm{ADF}$ and EDS cross-sections and for the first time. The validity of the two methods was verified for a $\sim 5 \mathrm{~nm}$ nanoparticle and future acquisition and analysis approaches are proposed utilising multiframe spectroscopy.

\section{Materials and Methods.}

\subsection{Calibration standards and acquisition methods.}

In the method presented here, the key to measuring a spectroscopic cross-section is having a sample with varying thickness. A fraction of the scattered intensity from the sample and electron probe interaction is recorded as a function of various sample thicknesses. From this, a single atom partial scattering cross-section can be calculated (Craven et al., 2016; Macarthur et al., 2015). Here we used nanoparticles of varying size and atom probe needle samples for comparison and cross-section measurements.

The pure element Pt nanoparticles used for the partial cross-section measurement were a commercially produced oxygen reduction reaction catalyst from Johnson Matthey. The nanoparticles were supported on carbon black and have a median size range of $3.25 \mathrm{~nm}$. The same batch of nanoparticles were used in a previous study atom counting study, (Aarons et al., 2017), where one of the findings linked the number of atoms in the nanoparticles to the nanoparticle diameter to 
demonstrate the accuracy of ADF-based atom counting. A Pt EELS partial cross-section was not measured for the Pt nanoparticles due to difficulties in obtaining sufficient signal from the available edges for small samples. Additionally, Co EELS partial cross-sections from nanoparticles were also omitted from the study due to the difficulty in synthesising pure element Co nanoparticles due to oxidation.

The carbon-supported Pt nanoparticle sample was crushed using mortar and pestle, drop-cast on a carbon grid, and mounted onto a JEOL Be double-tilt holder for the partial cross-section measurements. For the single EDS detector microscope, the holder was tilted towards the EDS detector by $20^{\circ}$ in the $+x$ direction. The EDS detector in this microscope is placed at right angles (on the right-hand side) with respect to the sample holder. Whereas, for the double EDS detector microscope, the holder was tilted by $15^{\circ}$ in in the $+\mathrm{x}$ and $+\mathrm{y}$ directions, as the second detector is front placed. Nanoparticles from the centre of the copper grid were chosen for analysis to minimise shadowing. Additionally, the Be holder varied for the single and double EDS systems. For the single EDS detector microscope, the holder has a machine-thinned side for optimal X-ray collection at $20^{\circ}$ $+x$ tilt. Whereas, the Be holder on the double EDS detector microscope is machined even more thinly on both the front and the side for optimal X-ray collection.

As an alternative, to measuring both the Pt and Co spectroscopic EDS and EELS partial crosssections, a needle calibration standard was prepared using a Zeiss Nvision 40 dual-beam Focused Ion Beam (FIB) instrument. The Pt and Co needle standards were prepared by electropolishing wire and then finally sharpened using FIB (Miller and Russell, 2007). The needles were mounted onto a Fischione 2050 tomography holder for the experiment. With this approach both Pt and Co cross sections can be measured with sufficient EDS and EELS signals. The maximum thickness of the needle can be measured by tilting the needle by $90^{\circ}$. However, to measure the entire needle surface thickness, an accurate measurement of the mean free path $(\lambda)$ is required. Using a method proposed by (Craven et al., 2016) the mean free path was measured and compared to the Malis and Iakoubovskii parameterisations (Iakoubovskii et al., 2008; Malis et al., 1988).

Table 1 summarises the signals obtained using the two calibration standards. The spectroscopic partial cross-sections were obtained using methods described here and building upon work from (Craven et al., 2016; Macarthur et al., 2015). For Pt, the $L \alpha, L \beta, M \alpha+M \beta$ and their total partial cross-sections were measured. Whereas for $\mathrm{Co}$, the $\mathrm{K} \alpha, \mathrm{K} \beta$ and their total partial cross-sections were measured.

The Pt EDS partial cross-section measurements between the needle and nanoparticle methods were compared for consistency. Then, atom counts from the measured Pt EDS partial cross-section were compared to an independent ADF quantification for an on-axis pure Pt nanoparticle. The ADF quantification was performed using Voronoi integration and simulation matching (Jones et al., 2014). In this approach, the intensity around an atomic column is integrated by segmenting the atomic columns into 'Voronoi' cells based on the distance between neighbouring atomic columns (Rosenauer et al., 2011). The integrated intensity from the Voronoi cell is converted into a scattering cross-section and the number of atoms in each atomic column are obtained by simulation matching. Similarly, the EDS quantification also used Voronoi integration, but the atom counts were obtained by dividing the total cross-section of a given column by the single atom Pt partial cross-section.

\begin{tabular}{c|lllll}
\multicolumn{1}{|c|}{} & \multicolumn{3}{c}{ Nanoparticle } & \multicolumn{3}{c}{ Needle } \\
\hline & & Pt & Co & Pt & Co \\
Partial & EDS & $\checkmark$ & & $\checkmark$ & $\checkmark$ \\
cross- & EELS & & & $\checkmark$ & $\checkmark$ \\
section $(\sigma)$ & & & & &
\end{tabular}

Table 1 summary of the spectroscopic calibration standards and the partial cross-sections obtained. 
Experimental data was acquired on a JEOL ARM 200CF microscope operated at 200kV acceleration voltage with a probe size of $\sim 0.8-1 \AA$. For the EDS partial cross-section benchmark test, EDS partial cross-sections were measured using nanoparticle standards on a single, and double EDS detector system. The acquisition parameters for the different samples are listed in Table 2, where the probe convergence $\alpha$, EELS collection angle $\beta$ and ADF detector collection angles were directly measured, with $99 \%$ accuracy, using a method from (Jones et al., 2017). Additionally, ray tracing and projector lens optimisation was performed, with the help of JEOL, using a method proposed by (Craven et al., 2017). This ensures that the effect of chromatic aberrations from the electrons entering the GIF are minimised.

The probe current was calibrated using the EELS drift tube for each spot size and microscope indicated emission. For the Pt and Co needle in Table 2, the needle experiments were performed at various beam currents ranging from $74-412 \mathrm{pA}$. As both microscopes in this experiment were equipped with cold field emission guns, the probe current in such systems would gradually decay during the experiment. For the microscopes used in this experiment, the current decayed by about $1 \%$ - $2 \%$ every 15 minutes. Thus, the beam current was constantly monitored by noting the indicated emission throughout the experiment day.

\begin{tabular}{|c|c|c|c|c|c|c|}
\hline Sample & $\begin{array}{c}\text { Experiment } \\
\text { probe current } \\
(p A)\end{array}$ & $\begin{array}{c}\text { Approximate } \\
\text { dose } \\
\left(e^{-} \AA^{2}\right)\end{array}$ & $\begin{array}{c}\text { Pixel } \\
\text { dwell time }\end{array}$ & $\begin{array}{c}\text { EELS collection } \\
\text { angle } \beta \text { (mrad) }\end{array}$ & $\begin{array}{c}\text { Probe } \\
\text { convergence } \\
\text { angle } \alpha \text { (mrad) }\end{array}$ & $\begin{array}{c}\text { ADF detector } \\
\text { collection } \\
\text { angle }(\text { mrad })\end{array}$ \\
\hline Pt Needle & $74-412$ & $2.9-4.7 \times 10^{7}$ & $0.02 \mathrm{~s}$ & 37.94 & 22.48 & $40.96-90.52$ \\
\hline Co Needle & $74-397$ & $2.9-4.5 \times 10^{7}$ & $0.02 \mathrm{~s}$ & 37.94 & 22.48 & $40.96-90.52$ \\
\hline $\begin{array}{l}\text { Pt off-axis NP } \\
1 \text { EDS detector }\end{array}$ & 186 & $3.7 \times 10^{6}$ & $0.001 \mathrm{~s}$ & N/A & 22.48 & $50.39-242.03$ \\
\hline $\begin{array}{c}\text { Pt off-axis NP } \\
2 \text { EDS detectors }\end{array}$ & 67 & $1.34 \times 10^{6}$ & $0.001 \mathrm{~s}$ & N/A & 22 & $\sim 50-240$ \\
\hline Pt on-axis $N P$ & 85 & $8.47 \times 10^{4}$ & $50 \mu \mathrm{s}$ & N/A & 22.48 & $50.39-242.03$ \\
\hline
\end{tabular}

Table 2 summary of acquisition parameters used during the experiment. $\mathrm{NP}=$ nanoparticle.

\subsection{Spectroscopic quantification theory.}

In the same way that the ADF scattering cross-section can be calculated from the image intensity (E et al., 2013; Retsky, 1974), it is possible to calculate an EDS (Macarthur et al., 2015) and EELS (Egerton, 2011; Hofer, 1991) partial scattering cross-section. The scattering cross-section has been shown to be robust to several parameters such as tilt, defocus, field of view, source size and astigmatism(E et al., 2013).

For thin samples such as nanoparticles, where absorption, fluorescence, multiple scattering and channelling (Nellist, 2011) can be neglected, the EDS partial scattering cross-section for a single atom of element $x$ is given by (Macarthur et al., 2015):

$$
\sigma_{x}^{E D S}=\frac{I_{x} e}{i \tau n_{x} t}
$$

Where $n_{x}$ is the volumetric number density of the elemental species being detected, $t$ is the sample thickness, $I_{x}$ is the raw x-ray counts detected from the sample, $i$ is the probe current, $\tau$ is the exposure time and $e$ is the electronic charge. Here we have assumed that the sample in projection can be regarded as homogeneous on the scale of the illuminating probe. For atomic-resolution studies, an integration of the counts over a Voronoi cell in the EDX map gives a partial cross-section for that column, by analogy with the approach for ADF cross-sections (E et al., 2013). For comparison, the popularly used $\zeta$-factor method defined by (Watanabe et al., 2003) is:

$$
\zeta_{x}^{E D S}=\frac{\rho t}{I_{x}} \times C_{x} D_{e}
$$


Where $I_{x}$ is the raw x-ray counts, $\rho$ is the sample density in $\mathrm{kg} / \mathrm{m}^{3}, t$ is the sample thickness, $C_{x}$ is the weight fraction of element $x$. And $D_{e}$ is the total number of electrons the sample is exposed to during the experiment, expressed by equation 3 .

$$
D_{e}=i \tau / e \quad 3
$$

In equation 2, the definition of sample density in the zeta-factor approach is in $\mathrm{kg} / \mathrm{m}^{3}$ which makes absolute quantification of nanoparticles, in terms of "number of atoms", cumbersome. Nonetheless, partial cross-section is very similar to the $\zeta$-factor and can be equated to it using equation 4 . Where $\mathrm{M}$ is the molar mass (in $\mathrm{kg} / \mathrm{mol}$ ) and N_A is Avogadro's constant.

$$
\sigma_{x}^{E D S}=\frac{M}{N_{A} \zeta_{x}}
$$

Similarly, the EELS partial cross-section can be obtained by normalisation of the core-edge intensity by the zero-loss peak $I_{0}$, which is approximated as the total incoming electron beam. In the single scattering case, the EELS partial cross-section for element $x$ is given by (Egerton, 2011):

$$
\sigma_{x}^{E E L S}=\frac{I_{x}}{I_{0} n_{x} t}
$$

The first attempt at linking EDS and EELS signals was by (Kothleitner et al., 2014), where the zeta factor and the EELS cross-section were linked to their respective measured intensities as follows:

$$
\begin{gathered}
\sigma_{x}^{E E L S}=\left(\frac{I_{x}^{E E L S}}{I_{0}} \times \frac{i \tau e^{-1}}{I_{x}^{E D S}}\right) \frac{A_{r}}{N_{A} \zeta_{x}} \\
\sigma_{x}^{E E L S} \times \zeta_{x}\left(\frac{N_{A}}{A_{r}} \times \frac{I_{0}}{i \tau e^{-1}}\right)=\frac{I_{x}^{E E L S}}{I_{x}^{E D S}}
\end{gathered}
$$

Once again, this expression is rather cumbersome to deal with, as a number density conversion from $\mathrm{kg} / \mathrm{m}^{3}$ to atoms $/ \mathrm{m}^{3}$ is required. This can be simplified by representing the EDS signal in scattering cross-section notation resulting in:

$$
\sigma_{x}^{E E L S}=\left(\frac{I_{x}^{E E L S}}{I_{0}} \times \frac{i \tau e^{-1}}{I_{x}^{E D S}}\right) \sigma_{x}^{E D S}
$$

For thin samples, if $I_{0}$ and $i \tau / e$ are represented by the same total number of electrons interacting with the sample (derived from the full beam current), $I_{0}$ and $i \tau / e$ cancel out. Thus, an obvious relationship between the scattering cross-section and the measured intensity is obtained:

$$
\frac{\sigma_{x}^{E E L S}}{\sigma_{x}^{E D S}}=\frac{I_{x}^{E E L S}}{I_{x}^{E D S}}
$$

The choice of $I_{0}$ is discussed in more detail in the discussion section of the paper. For thin samples $<10 \mathrm{~nm}$, equation 8 allows for an easy switch between the EDS and EELS signal intensities using a linear relationship which is a ratio of their partial scattering cross-sections. 
By combining spectroscopic data in this manner, processing of multivariate datasets becomes much easier and increases data throughput when analysis several nanoparticles. In the case of bimetallic PtCo nanoparticles, signals can be collected simultaneously for a hybrid elemental map depending on the difficulty of processing a spectroscopic edge in either method. For example, high energy EELS edges such as the Pt M5 - M4 have low signal to background ratio, making it difficult to process. This element could alternatively be quantified using EDS, where the Pt L and $\mathrm{M}$ signals are easier to acquire. Whereas, the Co $\mathrm{L}_{2,3}$ edges could be quantified using EELS because of easier acquisition and well defined white lines.

\subsection{Experimental Quantification procedure.}

Figure 1 summarises the quantification procedure that was used for the needle and nanoparticle standards with the general workflow for each technique. The needle method determined the partial cross-section from the sample thickness whereas, the nanoparticle method determines the partial cross-section from the number of atoms. The key to get both methods to converge to obtain similar results is in determining the thickness and number of atoms accurately. Each processing step is discussed in more detail in the upcoming subsections.

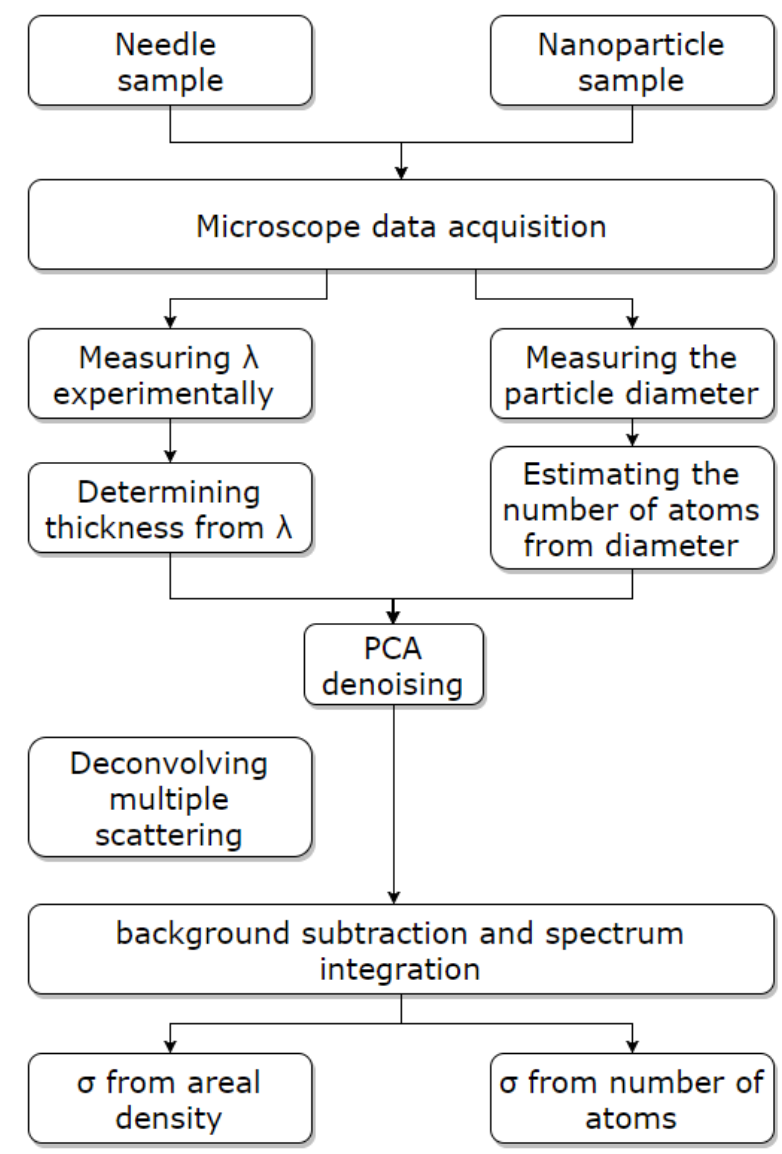

Figure 1 the spectroscopic data acquisition workflow for the needle and nanoparticle standards, from microscope acquisition and data analysis to partial cross-section measurements.

\subsubsection{Background subtraction and Integration parameters.}

Table 3 shows the spectral integration and background-subtraction windows that were used, these windows were applied consistently throughout both the needle and nanoparticle analyses. Ensuring consistency between the partial cross-section calculations also allowed for benchmark comparison between microscopes. 
For the EDS quantification a two-window background subtraction was used. For integrating the EDS signal, the integral limits were defined conventionally as $1.2 \times$ full width half maximum of the peak. For the EELS background subtraction, an inverse power-law subtraction was used. Modelling the signal peaks and correlating the fitting coefficients to the peak integral was also considered, but this would mean that the partial cross-section obtained would be defined with a new fitting reference. Additionally, the time taken to fit a model over several spectra using the currently established algorithm in Hyperspy is computationally intensive for a large spectrum image. Future implementations within Hyperspy, such as Smart Adaptive Multi-Dimensional Fitting (SAMFire) (Ostaševičius et al., 2016), aim to rectify this.

The choice of using a narrow $30 \mathrm{eV}$ EELS Co integration window in Table 3 was determined with PtCo nanoparticle experimental conditions in mind, which will be explored in a future study. To sample composition variation across the nanoparticle accurately, the EELS spectra should be recorded at low enough doses without causing significant structural damage to the nanoparticle. Sometimes in this situation, a smaller integration window is more favourable due to low signal to noise (SNR) (Kothleitner and Hofer, 1998). For example, a narrow 30eV Co signal integration window provides better SNR compared to a larger integration window. The larger integration window in this instance would worsen the SNR as the post edge signal is weak.

\begin{tabular}{|c|c|c|c|c|}
\hline & & $\begin{array}{l}\text { Background } \\
\text { windows }(\text { keV })\end{array}$ & $\begin{array}{l}\text { Integration } \\
\text { windows }(\text { keV })\end{array}$ & $\begin{array}{l}\text { Theoretical peak } \\
\text { ledge maxima (keV) }\end{array}$ \\
\hline \multirow[t]{5}{*}{$E D S$} & Co Ka & $6.51-6.65,7.21-7.35$ & $6.65-7.21$ & 6.93 \\
\hline & Со K $\beta$ & 7.21-7.36, 7.94-8.09 & $7.40-7.86$ & 7.65 \\
\hline & Pt La & $9.08-9.15,9.65-9.72$ & $9.15-9.65$ & 9.44 \\
\hline & Pt L $\beta$ & $10.64-10.71,11.43-11.50$ & $10.71-11.43$ & 11.07 \\
\hline & Pt M $\alpha+\mathbf{M} \beta$ & $1.77-1.85,2.40-2.49$ & $1.85-2.24$ & $2.05,2.12$ \\
\hline \multirow[t]{2}{*}{$E E L S$} & Co L3 \& L2 & $60 \mathrm{eV}$ width before peak & $30 \mathrm{eV}$ width & $779 \mathrm{eV}, 794 \mathrm{eV}$ \\
\hline & Pt M5 -M4 & $150 \mathrm{eV}$ width before peak & $300 \mathrm{eV}$ width & $2122 \mathrm{eV}, 2202 \mathrm{eV}$ \\
\hline
\end{tabular}

Table 3 The integration and background subtraction windows for the EDS and EELS partial crosssections.

\subsubsection{Needle quantification.}

For the data acquisition step in Figure 1, an appropriate $0^{\circ}$ tilt for the needle was determined and the spectroscopic data was recorded. Care was taken to ensure that the needle was tilted away from a major zone axis by observing the Kikuchi lines. To measure the thickness across the needle, the mean free path must be determined accurately. For further details on how this is performed, the reader should refer to the publication by (Craven et al., 2016). The advantage of this approach is that a pixelwise thickness determination can be obtained for the sample. The following paragraph summarises this procedure for context.

A low loss Spectrum Image (SI) of the needle was recorded and the maximum $t / \lambda$ was plotted as a function of axial position from a determined $0^{\circ}$ needle tilt, Figure $2 \mathrm{a}$. The needle was then tilted by $90^{\circ}$ so that the maximum thickness $(t)$ could be determined by image thresholding, Figure $2 \mathrm{~b}$. Following subsequent alignment of the $0^{\circ}$ and $90^{\circ}$ needle tips, the $t / \lambda$ profile followed the absolute thickness profile very closely for most axial positions except at the tip due to oxidation, Figure 2c. Points where the $t / \lambda$ was less than 1 and followed the absolute thickness profile closely were used for the $\lambda$ calculation. The $\lambda$ was obtained from the gradient of a linear plot of $t^{2}$ against $(t / \lambda)^{2}$, Figure $2 \mathrm{~d}$. The thickness was then determined by multiplying $\lambda$ to the $t / \lambda$ map. 

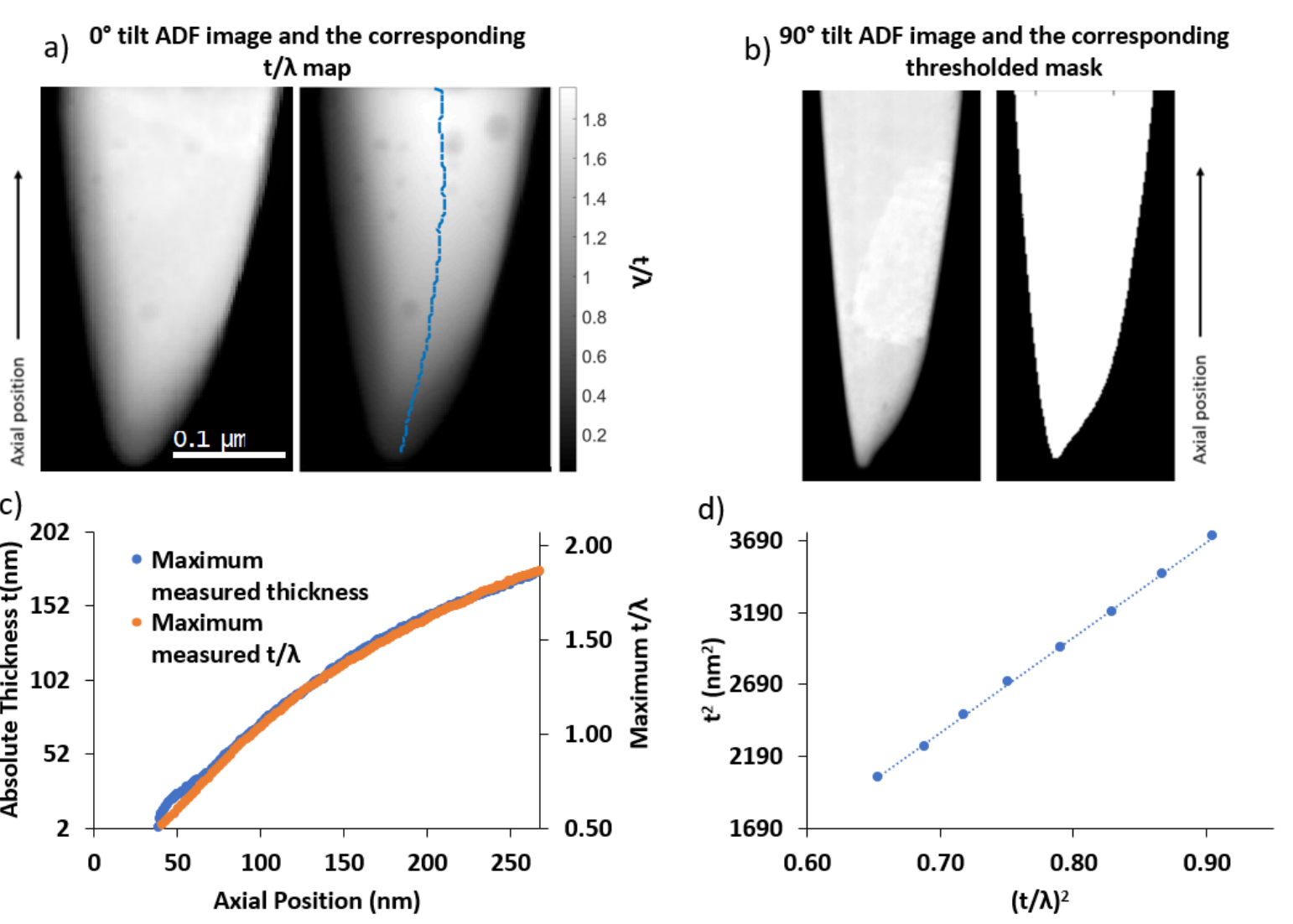

Figure 2 a) $0^{\circ}$ tilt ADF image with a corresponding $t / \lambda$ map, with the maximum $t / \lambda$ annotated by the blue line, for a Co needle, b) $90^{\circ} \mathrm{ADF}$ image of the needle with a thresholded mask that was used to determine the absolute thickness of the needle, c) plot of absolute thickness against maximum $t / \lambda$ for each axial position; and d) plot of $t^{2}$ against $(t / \lambda)^{2}$ with a linear fit from which the mean free path was measured.

The data for the spectroscopic partial cross-section measurements was recorded using a combination of spectrum images (SI) and linescans across the needle. Spectrum images were recorded with a step size of $\sim 2 \mathrm{~nm}$, whereas the linescans were recorded with a $\sim 0.5 \AA$ step size. The EELS partial crosssection was measured from the SI so that a broad range of thicknesses could be covered from the needle. The EDS partial cross-section was measured using finely sampled linescans with a $\sim 0.5 \AA$ step size. Due to the low X-ray yield, finely sampled linescans ensured that the spectrum could be binned appropriately while sufficiently sampling the sample morphological changes across the needle as the probe scanned the specimen.

It is also possible to collect a finely sampled SI map of the needle but due to the large field of view, the file size (hundreds of gigabytes) and time (several hours) required to record this data cube is not justified. In contrast, line scans and less finely sampled SI maps have a smaller file size and require less time to record. Acquiring spectra in this way also mitigates any afterglow effects that would affect the EELS dark reference correction and the partial cross-section measurements. Additionally, the EELS "bleed through" effect was addressed using a similar method proposed by (Bobynko et al., 2015).

The low-loss and the high-loss EELS spectra were binned, aligned and denoised using Hyperspy (de la Pena et al., 2017). Principal Component Analysis (PCA) was used for denoising and the processed spectra were checked to ensure that there were no artefacts from missing components. A comparison of the raw and denoised spectra for a selected pixel is shown in Figure 3. Since only the thin regions of the needle were analysed, deconvolution was not necessary. For the EDS fractionation in equation 
1, the probe current was measured using the EELS drift tube and for the EELS fractionation the zeroloss peak intensity in equation 5 was scaled using a CCD linearity test. The Pt and Co number densities were calculated geometrically using lattice parameters from (Davey, 1925) and (Owen and Jones, 1954) for Pt and Co respectively. The spectroscopic partial cross-sections were then calculated using the measured beam current, ZLP, number density and the sample thickness as shown by the flow chart, Figure 1.

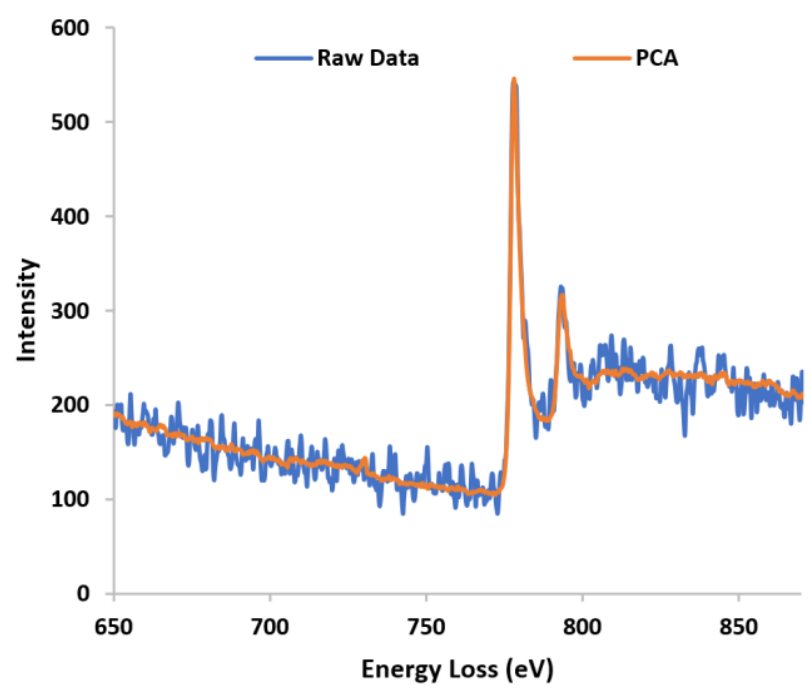

Figure 3 Comparison of the EELS spectra before and after PCA denoising from a $\sim 30 \mathrm{~nm}$ thickness region of the Co needle.

\subsubsection{Nanoparticle quantification.}

For the nanoparticle partial cross-section calibration, spectrum images for the particles were taken. An area on the specimen grid containing up to 12 non-overlapping nanoparticles was selected and the SI was taken in a burst of 4 sequential frames so that particle drift could be observed (Jones et al., 2018).

To measure the nanoparticle diameter and number of atoms, the ADF image in each frame was thresholded and segmented using a watershed transform, Figure 4. The diameter was estimated by equating the segment area of the nanoparticle to the area of a circle and averaged across the frames. The number of atoms for each nanoparticle was determined by estimating the diameter of the nanoparticles as a sphere with an fcc lattice. This approximation was validated in a previous study on the same nanoparticles were the number of atoms were found to increase with $\sim r^{3}$, with a number density pre-factor matching bulk Pt (Aarons et al., 2017).

The EDS intensities within each mask were summed as the collective nanoparticle intensity and normalised by the intensity of the incident electron beam (i.e. $I_{x} / i \tau e^{-1}$ ). Then fractional intensity was multiplied by the pixel area to obtain a total nanoparticle cross-section in units of barns. From this, the single-atom partial EDS cross-section was obtained by dividing the total partial cross-section by the number of atoms in the nanoparticle. This method is analogous to the method used by (E et al., 2013) where the sum of the measured ADF intensity is multiplied by the pixel area to obtain a scattering cross-section. 


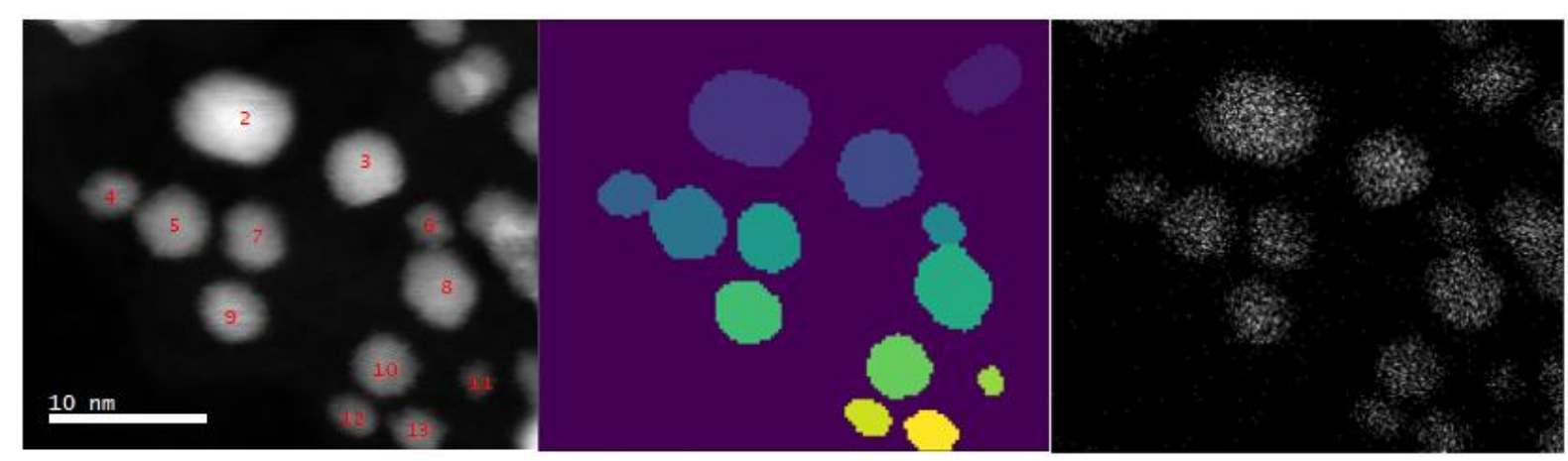

Figure 4 (left) size selected non-channelling Pt nanoparticles used for the partial cross-section measurements, (centre) the thresholded and segmented imaged that was used to mask the EDS spectrum and (right) the raw Pt EDS signal from each nanoparticle before binning.

\subsubsection{On-axis ADF and EDS nanoparticle quantification.}

For the final test of verifying the EDS partial cross-section obtained from standards, an on-axis $\mathrm{Pt}$ nanoparticle was atom counted using both EDS and ADF quantification techniques. In the ADF quantification approach, data was collected using multiframe acquisition and non-rigid aligned (Jones et al., 2015). To measure the ADF collection angles accurately and precisely, a recently developed calibration technique was employed (Jones et al., 2017). The column peak intensities from the aligned data were Voronoi integrated and quantified using simulation matching (Jones et al., 2014; Rosenauer et al., 2009). The ADF image simulations were performed using the $\mu$ STEM software (Allen et al., 2014).

Similarly, the EDS signal was collected using a multi-pass approach (Jones et al., 2018), where the column peaks and Voronoi integration areas were obtained from the simultaneously acquired ADF image. The integrated EDS intensity was subsequently converted into atom counts using the single atom Pt partial cross-section calibration. Due to this, channelling was not considered and would contribute to the error by overestimating the number of atoms in the on-axis EDS quantification and is discussed later in the results section.

\section{Results and discussion}

Table 4 compares the measured experimental mean free path with the Malis and the Iakoubovskii parametrisations. For the convergence and collection angles used, the data shows good agreement with the Malis parameterisation, which is surprising. Analysis performed by (Craven et al., 2016) for high convergence and collection angles in modern aberration corrected STEMs also leans towards similar conclusions. This measurement shows that determining an accurate mean free path is key to obtaining an accurate partial cross-section measurement as choosing the wrong parameterisation could lead to errors of up to $20 \%$. However, it is still not clear as to which parameterization to use universally as it has been shown to depend on the collection angle and element in question (Zhang et al., 2012).

The number density of the needles was derived by assuming a fcc structure for both elements with their respective lattice parameters from (Davey, 1925) and (Owen and Jones, 1954). However, Co is also well known to have hop structure (Owen and Jones, 1954), this was also calculated, and the number density does not change significantly $\left(90.28\right.$ atoms $\left./ \mathrm{nm}^{3}\right)$ compared to the fcc structure shown in Table 4. Both $\mathrm{Co}$ and $\mathrm{Pt}$ number density values agree well with previous calculations by (Macarthur et al., 2015). 


\begin{tabular}{r|ll} 
& Pt & Co \\
\hline Density $\left(\right.$ atoms $\left./ \mathbf{n m}^{3}\right)$ & 66.41 & 90.16 \\
Malis mean free path $(\mathbf{n m})$ & 59.53 & 81.40 \\
Iakoubovskii mean free path $(\mathbf{n m})$ & 88.40 & 109.10 \\
Experimental mean free path $(\mathrm{nm})$ & 57.57 & 81.55
\end{tabular}

Table 4 the calculated and measured mean free path parameters for both $\mathrm{Pt}$ and Co needles. The mean free path agrees with at least one of the calculated parameterisations.

Once the number density and the mean free path is determined accurately, the thickness per binned pixel can be plotted against a "fraction of the incident beam", i.e. $I_{x} / i \tau e^{-1}$ for EDS and $I_{x} / I_{0}$ for EELS, using equations 1 and 5 respectively, Figure 5. This quantity represents the fraction of the incident beam that is scattered and gives rise to the detected spectroscopic signal. By representing the detected spectroscopic signal in terms of its fraction of the incident beam, a direct comparison of the signal efficiency can be made between EELS and EDS signals.

The $I_{0}$ EELS fractionation in Figure 5 was defined by the zero-loss peak from the low-loss spectrum. Fractionating the data in this manner and obtaining the partial cross-section has shown good agreement with calculated Hartree-Slater cross-sections in previous work by (Craven et al., 2016) Data points from the thinner regions of the needle $\sim<5 \mathrm{~nm}-10 \mathrm{~nm}$ were removed from the analysis because these regions would be dominated by oxide layers. Additionally, thicker regions of the needle which are more susceptible to errors from deconvolution and absorption effects were also removed. Only the most linear part of the dataset, from the thin regions, was used for the EELS partial crosssection measurement. 

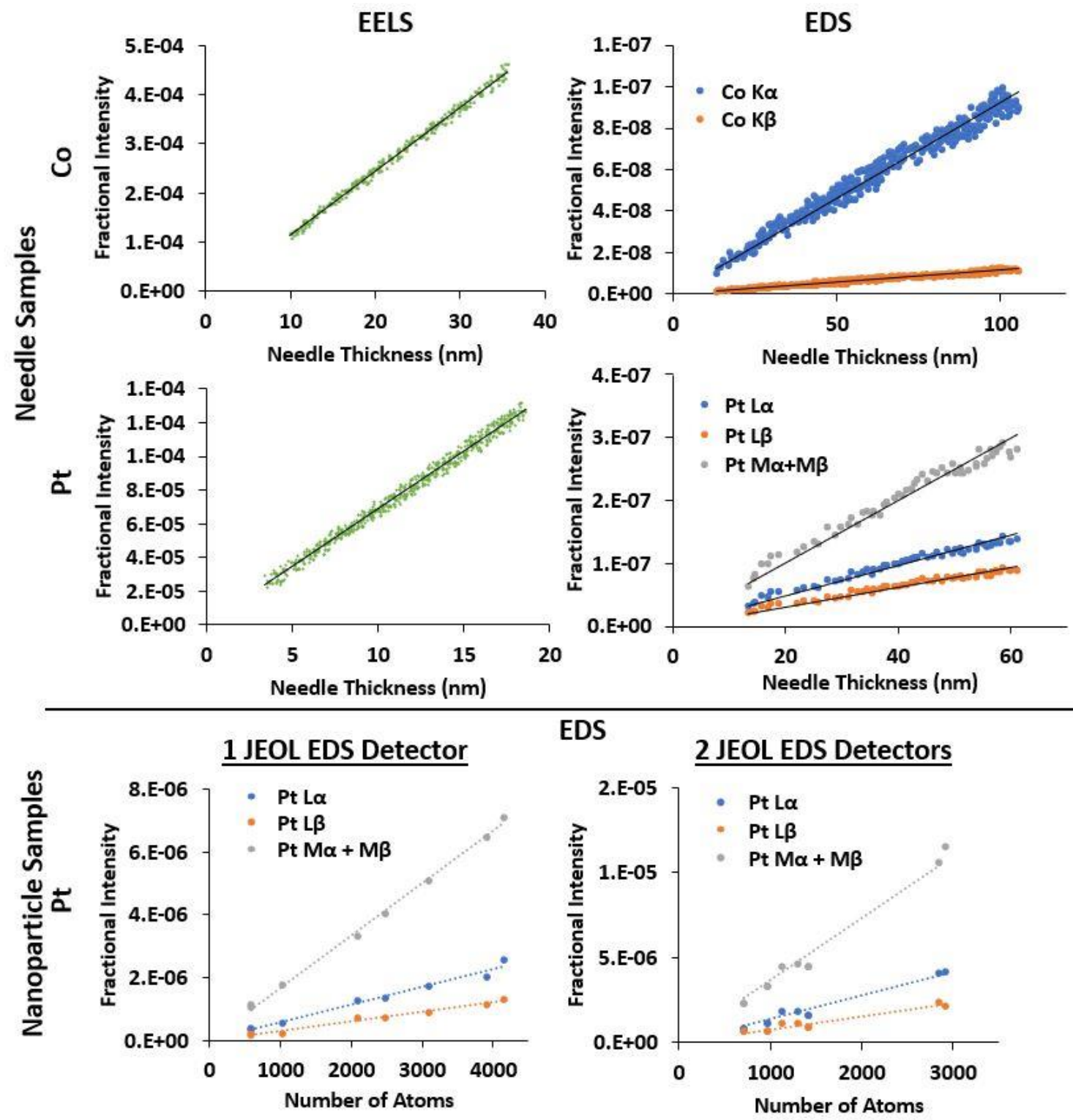

Figure 5 the EELS and EDS signals plotted in fractionalised intensity as a function of either the needle thickness or nanoparticle number. Normalising the spectroscopic intensity as fractional intensity allows for an easy visual comparison between the EELS and EDS signals for selected integration windows.

Various other "partial" cross-sections can also be defined using different definitions of $I_{0}$, Figure 6. For sufficiently thin samples, $<10 \mathrm{~nm}$, the full incoming beam, i.e. a ZLP in vacuum, can also be used. Fractionating the core-loss edge using a ZLP in vacuum for thin samples has two main advantages for nanoparticle quantification: 1), it does not require processing of the bleed through effect, thus a lowloss spectrum is not required and 2) it simplifies the processing of multivariate datasets as shown by equation 7 , thus increasing throughput for numerous datasets.

However, as the sample thickness increases, the elastic scattering intercepted by the EELS aperture increases. In this situation, it is more favourable to use the zero-loss peak from the low-loss spectrum as a first order compensation for the elastic scattering intercepted by the aperture. 


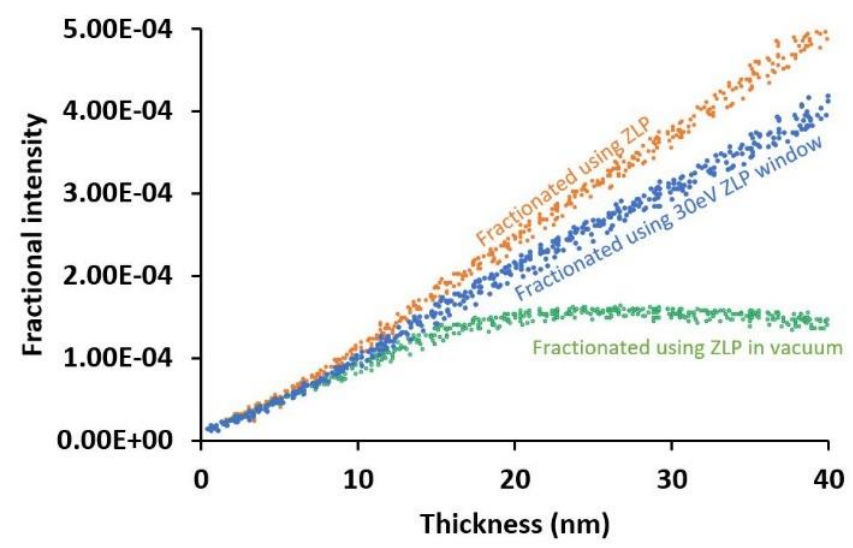

Figure 6 Fractional intensity $\left(I / I_{0}\right)$ from a Co $\mathrm{L}_{2,3}$ edge plotted as a function of thickness with different definitions of $I_{0}$ that can be used in equations 5 and 7 .

It is also possible to pick out background fitting and thickness systematic errors in Figures 5 and 6 . The plots should extrapolate to zero fractional intensity at lower thicknesses or number of atoms. This can be seen for all the plots hence the edge intensity integration and thickness determination were processed correctly.

A systematic error from the mean free path determination would also get washed out during the subsequent composition analysis (Craven et al., 2016) if the elemental quantification is done using the same mean free path and oxide removal process. In this situation, the gradient of the plot can still be used the determine a partial scattering cross-section, with an offset. Thus, the main EELS error contribution arises from the background modelling and integration, especially for very high energy loss edges such as Pt. This is where EDS acquisition would be more favourable to quantify the Pt composition within a nanoparticle sample. Finally, by using the slopes in Figure 5, the measured $\lambda$, and number density in Table 4, the needle EDS and EELS partial cross-sections were calculated. The calculated values are summarised in Table 5, where the needle and nanoparticle Pt EDS partial crosssections for the L-lines agree quite well and the $\mathrm{M}$ lines differ by more than $30 \%$. The discrepancy in the M-line quantification may be explained from the difficulty in the $\mathrm{M}$ line detection, which is more sensitive to absorption effects, which could cause significant errors in its quantification (Lábár and Salter, 1991).

Absorption effects due to the EDS signal acquisition and detector geometry were also considered, by measuring the EDS partial cross-section from the right and left halves of the needle. This seemed to have little impact on the overall $\mathrm{M}$ line partial cross-section. We did not expect this to be $\sim 30 \%$ and we are not sure what the cause of this is. This discrepancy reaffirms the need to cross-check crosssections measured using multiple standards, which are then used to quantify materials with different stoichiometries. However, the characteristic $\mathrm{K}$ and $\mathrm{L}$ lines agree well between the two samples and can be used for quantification.

As stated previously, the conversion of EDS partial cross-sections into units of barns, allows for an easier comparison with the simultaneously acquired EELS and ADF signals. For example, a direct comparison of the EDS signal shows that the EDS partial cross-section is 2 orders of magnitude smaller than the EELS and 6 orders of magnitude smaller than the collected ADF signal (typically $\mathrm{Mb}$ range) (Figure 8 and Table 5). For the two EDS detectors system, the Pt EDS partial cross-section is slightly more than double. The EDS partial cross-section gives a direct comparison of the efficiency between the two microscopes, which cannot be obtained from just measuring the solid angle. Here the stated nominal solid angles from JEOL would be up to $0.89 \mathrm{Sr}$ for a single EDS detector and $1.25 \mathrm{Sr}$ for a double EDS detector. It should also be noted that the microscope fitted with the double EDS detectors uses the current state of the art detector offered by JEOL and hence this comparison is not 
completely identical. The microscopes equipped with the double EDS system have an updated design compared to the single EDS detector system. Furthermore, as mentioned before, the Be holders between the microscopes were different and therefore would also contribute towards the partial crosssection measurements.

When the measured EELS partial cross-sections are compared to the Hartree Slater and hydrogenic models (Leapman et al., 1980), using the quantification tool in Digital Micrograph, they show good agreement with one another. A similar study conducted by (Craven et al., 2016) also shows very good agreement between measured and calculated EELS partial cross-sections. However, the aim here is not to obtain a perfect match between the measured and calculated partial cross-sections especially in the case for the high energy Pt edge. For a more thorough comparison the measured partial crosssection should be compared to a multislice (Allen et al., 2014) and DFT simulation (Tait et al., 2016).

A key point that should be noted when correlating the ADF, EDS and EELS signals is that if any of the microscope settings, such as detector placements (ADF, EDS or EELS) or post specimen optics (ADF and EELS) change drastically, the cross-sections in Table 5 would have to be remeasured. However, the calibration experiment is easy and software automation using Hyperspy (de la Pena et al., 2017), AtoMap (Nord et al., 2017), Absolute Integrator (Jones et al., 2014) and StatSTEM (De Backer et al., 2016) can in principle make this type of analysis routine.

\begin{tabular}{|c|c|c|c|c|c|c|}
\hline \multirow{10}{*}{ 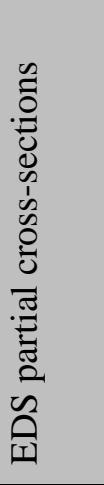 } & \multicolumn{6}{|c|}{ Pt EDS partial cross-section with a single EDS detector } \\
\hline & EDS lines & $\mathrm{L} \alpha$ & $\mathrm{L} \beta$ & $\mathrm{L} \alpha+\mathrm{L} \beta$ & $\mathrm{M} \alpha+\mathrm{M} \beta$ & $\mathrm{L}+\mathrm{M}$ \\
\hline & Needle & $5.55 b$ & $3.58 b$ & $9.13 b$ & $11.47 \mathrm{~b}$ & $20.60 b$ \\
\hline & Nanoparticles & $5.69 \mathrm{~b}$ & $3.05 b$ & $8.74 b$ & $16.67 \mathrm{~b}$ & $25.41 b$ \\
\hline & \multicolumn{6}{|c|}{ Pt EDS partial cross-section with double EDS detectors } \\
\hline & EDS lines & $\mathrm{L} \alpha$ & $\mathrm{L} \beta$ & $\mathrm{L} \alpha+\mathrm{L} \beta$ & $\mathrm{M} \alpha+\mathrm{M} \beta$ & $\mathrm{L}+\mathrm{M}$ \\
\hline & Nanoparticles & $13.68 \mathrm{~b}$ & $7.73 b$ & $21.41 \mathrm{~b}$ & $36.61 \mathrm{~b}$ & $58.02 \mathrm{~b}$ \\
\hline & \multicolumn{6}{|c|}{ Co EDS partial cross-section with a single EDS detector } \\
\hline & EDS lines & $\mathrm{K} \alpha$ & $\mathrm{K} \beta$ & $\mathrm{K} \alpha+\mathrm{K} \beta$ & & \\
\hline & Needle & $5.88 \mathrm{~b}$ & $0.76 \mathrm{~b}$ & $6.64 \mathrm{~b}$ & & \\
\hline \multirow{6}{*}{ 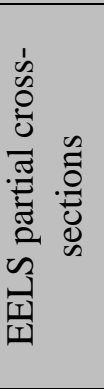 } & \multicolumn{6}{|c|}{ Pt EELS partial cross-section } \\
\hline & EELS edges & \multicolumn{5}{|c|}{ M5, M4 (300eV window) } \\
\hline & Needle & Measure & & $\begin{array}{r}\text { Calculated I } \\
\text { Calc }\end{array}$ & $\begin{array}{l}\text { e Slater: } \\
\text { l Hydrogenic: }\end{array}$ & $\begin{array}{l}1.26 \mathrm{~kb} \\
---\end{array}$ \\
\hline & \multicolumn{6}{|c|}{$\begin{array}{l}\text { Calculated Hydrogenic: } \\
\text { Co EELs partial cross-section }\end{array}$} \\
\hline & EELS edges & \multicolumn{5}{|c|}{ L3, L2 (30eV window) } \\
\hline & Needle & Measure & $3 \mathrm{~kb}$ & $\begin{array}{r}\text { Calcul } \\
\text { Calc }\end{array}$ & $\begin{array}{l}\text { Hartree Slater: } \\
\text { Hydrogenic: }\end{array}$ & $\begin{array}{l}0.88 \mathrm{~kb} \\
0.80 \mathrm{~kb}\end{array}$ \\
\hline
\end{tabular}

Table 5 Summary of the EDS and EELS single atom partial cross-sections (in barns) obtained from the needle and nanoparticle calibration standards. The standard \% errors are provided in Table 6 in the error analysis section.

The measured EDS cross-sections can now be used to provide a method for counting of the number of atoms in a nanoparticle as an alternative method to using ADF intensities. Although the lower crosssection will lead to lower precision, EDS does offer the potential of element specificity. Here the L + M line partial cross-sections from the nanoparticle standards were used to quantify an on-axis nanoparticle. Even though the needle and nanoparticle $M$ lines have a discrepancy, the $M$ line should be consistent between nanoparticles and can be exploited to increase the total $\mathrm{x}$-ray count. Combining $\mathrm{x}$-ray lines in this manner should be done with care, but it is necessary for thin samples to increase counting statistics. Finally, the nanoparticle EDS atom counts were compared to the atom counts obtained from the ADF quantification, Figure 7. The images in Figure 7 serve a dual purpose, the ADF images show the damage caused by the electron beam before and after ADF + EDS acquisition 
using the settings listed in Table 2. The column peaks are then overlaid with atom numbers from the ADF quantification on the left image and the EDS quantification on the right image. The total number of atoms between the two quantification techniques are within $10 \%$ agreement with one another. It should also be noted that the EDS quantification does not take channelling into account and is therefore expected to overestimate the number of atoms slightly (Allen et al., 2012; MacArthur et al., 2017). If channelling were considered, then the EDS partial cross-section would vary monotonically by atomic column thickness in a similar manner to the ADF cross-section, Figure 8.

Additionally, the edge atoms in the EDS quantification seem to have been overestimated significantly when compared to their ADF quantified counterparts. This may be attributed to the sequential multipass scan where the nanoparticle may have rotated and restructured over the acquisition period. The error bar on the EDS partial cross-section is also significantly higher than the ADF one. From Poisson-limited $(\sqrt{\mathrm{N}})$ counting statistics this error can be up to \pm 3 atoms (Macarthur et al., 2015) for the EDS quantification plus the errors arising from a non-channelling approximation. For the EDS quantification nanoparticle in Figure 7, the error in each atomic column ranges between 2 and 3 atoms depending on the integrated EDS intensity per Voronoi cell. None the less, the total EDS quantification is accurate within $10 \%$ for nanoparticles between $2 \mathrm{~nm}$ and $5 \mathrm{~nm}$, but the atom counting precision on a column by column basis is quite poor as observed in Figure 7. To improve upon this in the future, we aim to employ a multiframe SI acquisition routine, recently developed at the University of Oxford (Jones et al., 2018).
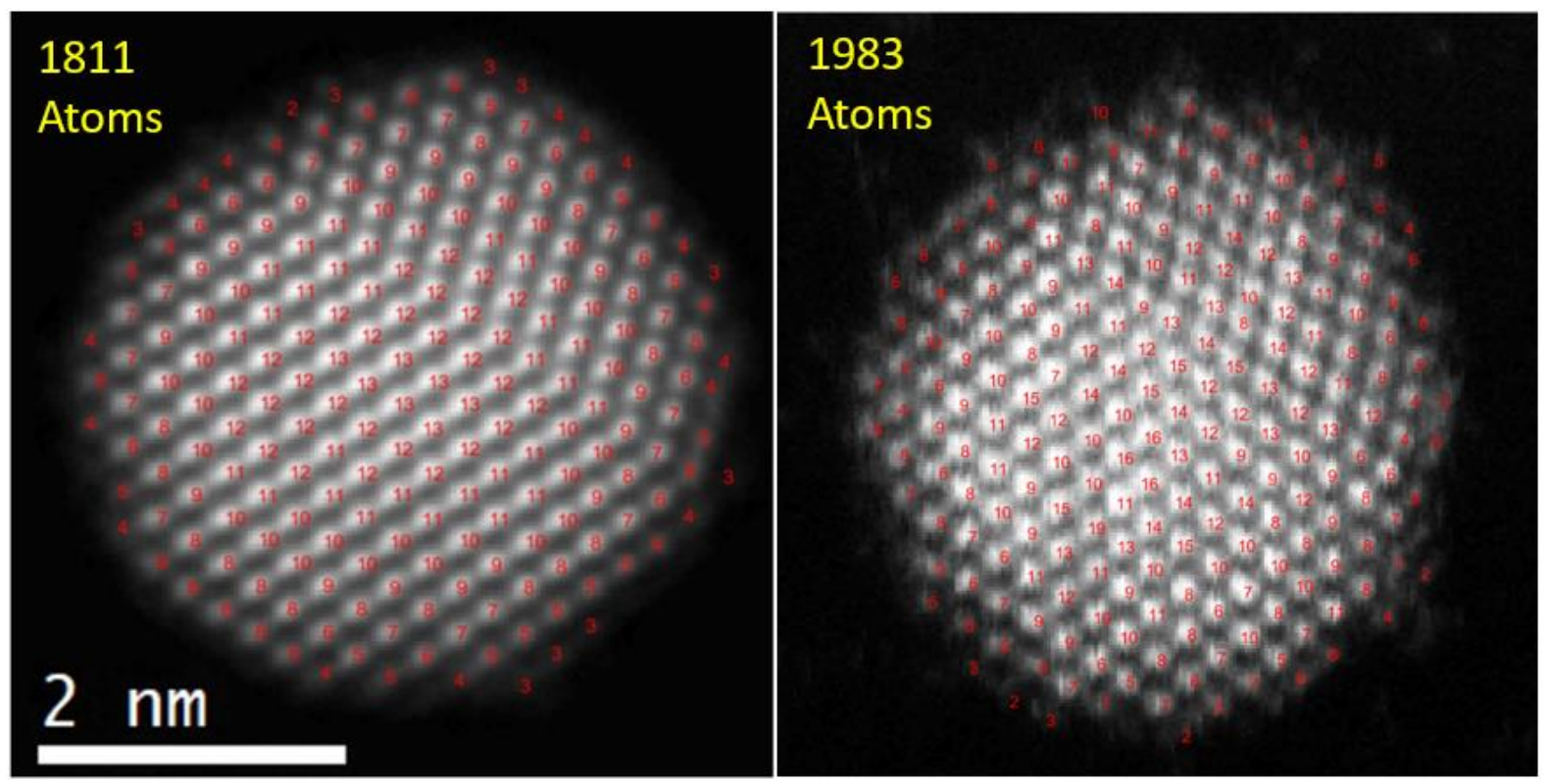

Figure 7 A comparison between the ADF(left) and the EDS(right) atom counts for an on-axis pure Pt nanoparticle for each atomic column, obtained from voronoi integration. The Left and right images also display the before and after effects of a SI acquisition at $\sim 85 \mathrm{pA}$ beam current and a $50 \mu \mathrm{s} \times 60$ frames pixel dwell time. 


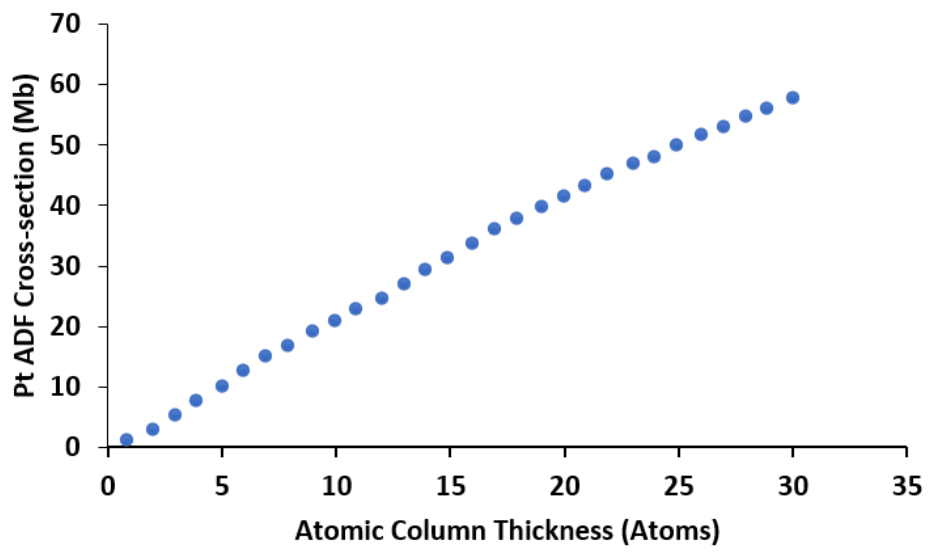

Figure 8 the $\mu$ STEM simulation library used to quantify the on-axis ADF nanoparticle. The scattering cross-section (in mega-barns, Mb) per atomic column varies monotonically with column thickness due to channelling.

\section{Error analysis}

\subsection{EDS partial cross-section error propagation for a single measurement}

The error analysis performed here is very similar to the $\zeta$-factor method proposed by (Watanabe and Williams, 2006). Here the error for a function $f$, with $n$ uncorrelated variables (i.e. $f=f\left(p_{1}, p_{2}, \ldots, p_{n}\right)$ ) and deviation $d$ can be given as

$$
d_{f}^{2}=\sum_{j=1}^{n}\left(\frac{\partial f}{\partial p_{i}}\right)^{2} d_{p_{j}}^{2}
$$

For the needle samples, in the case of the EDS partial cross-section, the major error contributors from equation 1 would be the number density $(n)$, thickness $(t)$ and the measured intensity $(I)$. The minor errors would be attributed to: the probe current to calculate the dose, giving a relative error of $\sim 0.5 \%$ and the pixel dwell time $(\tau)$ with a negligible contribution (Macarthur et al., 2015). Therefore, the error $\left(\Delta \sigma_{E D S}\right)$ from a single EDS partial cross-section $\left(\sigma_{\mathrm{EDS}}\right)$ can be derived similarly from(Watanabe and Williams, 2006) using equations 1 and 9 as:

$$
\Delta \sigma_{E D S}=\sigma_{E D S} \sqrt{\left[\left(\frac{\Delta n}{n}\right)^{2}+\left(\frac{\Delta t}{t}\right)^{2}+\left(\frac{\Delta I}{I}\right)^{2}\right]}
$$

Where $\Delta n, \Delta t$, and $\Delta I$, indicate errors in $n, t$, and $I$ respectively. Here the error in $n$ can be negligible if the analysis is performed from the bulk area of the needle to negate surface-oxide effects and the sample stoichiometry is well known. The $\Delta I$ term can have a $\sqrt{I}$ error if the signal obeys a Poisson distribution (Thompson, 2001; Watanabe and Williams, 2006). The $\Delta t$ term can be derived from the variance of several mean free path measurements.

The errors from equation 10 can be visualised by plotting their error bars and the corresponding partial cross-section as a function of thickness and nominal intensity, Figure 9. Both the Pt and Co errors bars decrease as the thickness of the sample increases up to a certain thickness, after which the portion of the error contribution from $\sqrt{I}$ decreases. 

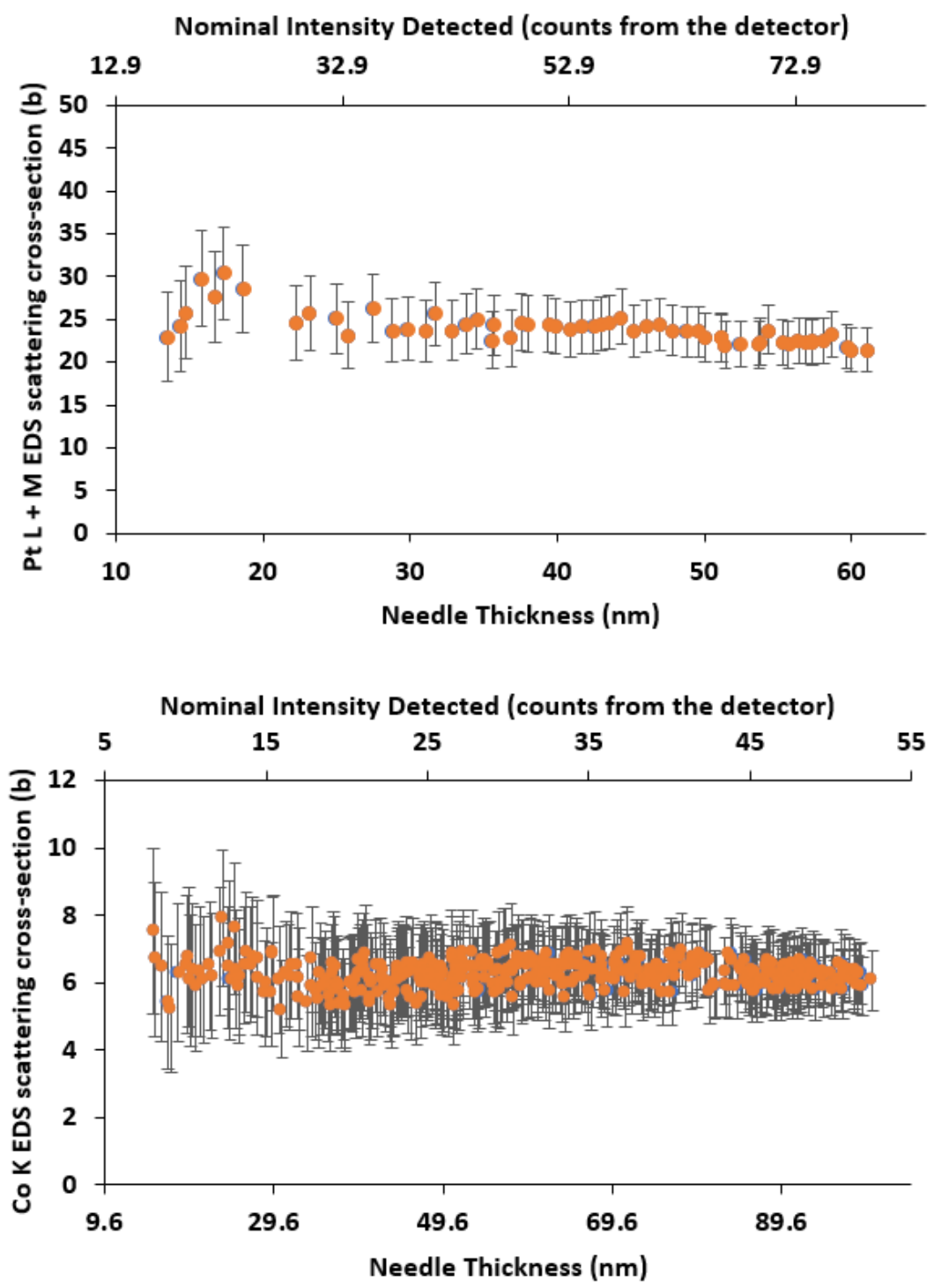

Figure 9 The single measurement error in the Pt and Co total EDS partial cross-sections as a function of thickness and nominal intensity (0.001s pixel dwell time, $\sim 74 \mathrm{pA}$ beam current) from the line scans in Figure 5.

Similarly, for the nanoparticles, the EDS partial cross-section error bars reduce with increasing number of atoms, Figure 10. The largest contributions to the error originate from counting statistics. After a certain nanoparticle size, $>2000$ atoms at the given dose, the $\sqrt{I}$ error becomes more consistent. At larger thicknesses, an increasing portion of the error is dominated by systematic errors such as the magnification calibration. The pixel area errors can vary by up to $\pm 4 \%$ (Macarthur et al., 2015 ) and depends on the magnification calibration. The error in the number of atoms determined for a nanoparticle depends on the thresholding mask resulting in a $\pm 5 \%$ deviation. 


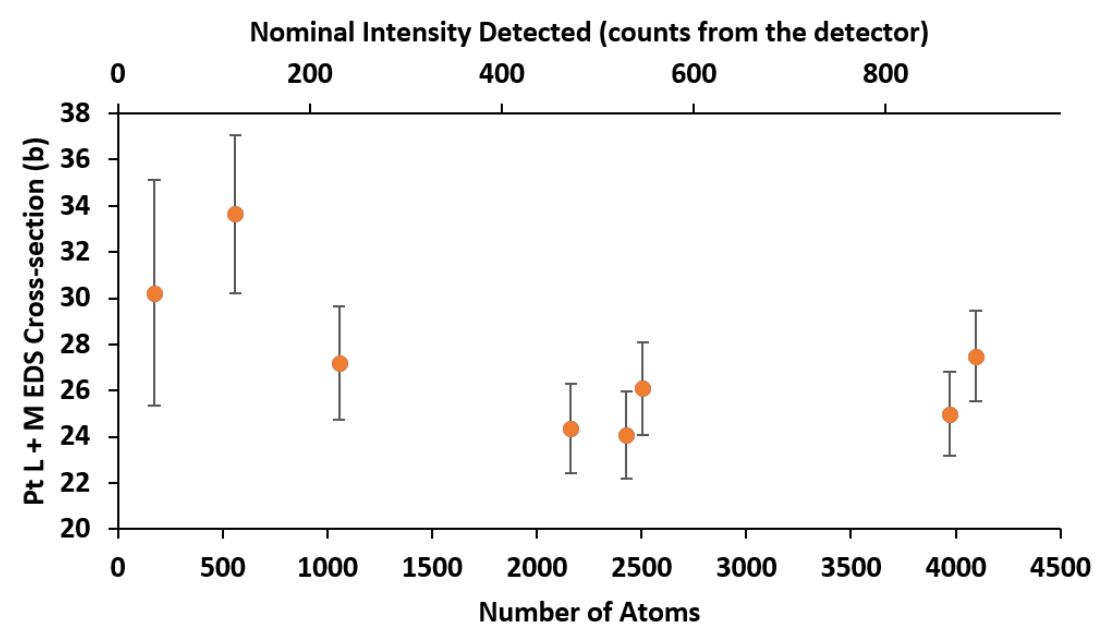

Figure 10, Single measurement error in the Pt tot EDS partial cross-section as a function of number of atoms and nominal intensity for a single EDS detector (0.001s pixel dwell time, $\sim 186 \mathrm{pA}$ beam current).

Since the dominant error propagator for nanoparticles is the counting statistics, the percentage error from this can be plotted as a function of thickness. Figure 11 shows this representation for a selected dose of $\sim 74 \mathrm{pA}$ from the $\mathrm{Pt}$ and Co needle total partial cross-section measurements. The error is clearly dose dependent and therefore limited by counting statistics. However, one should take sample damage into consideration as well. A delicate balance between dose and sample damage is required to achieve enough counting statistics without altering the sample.
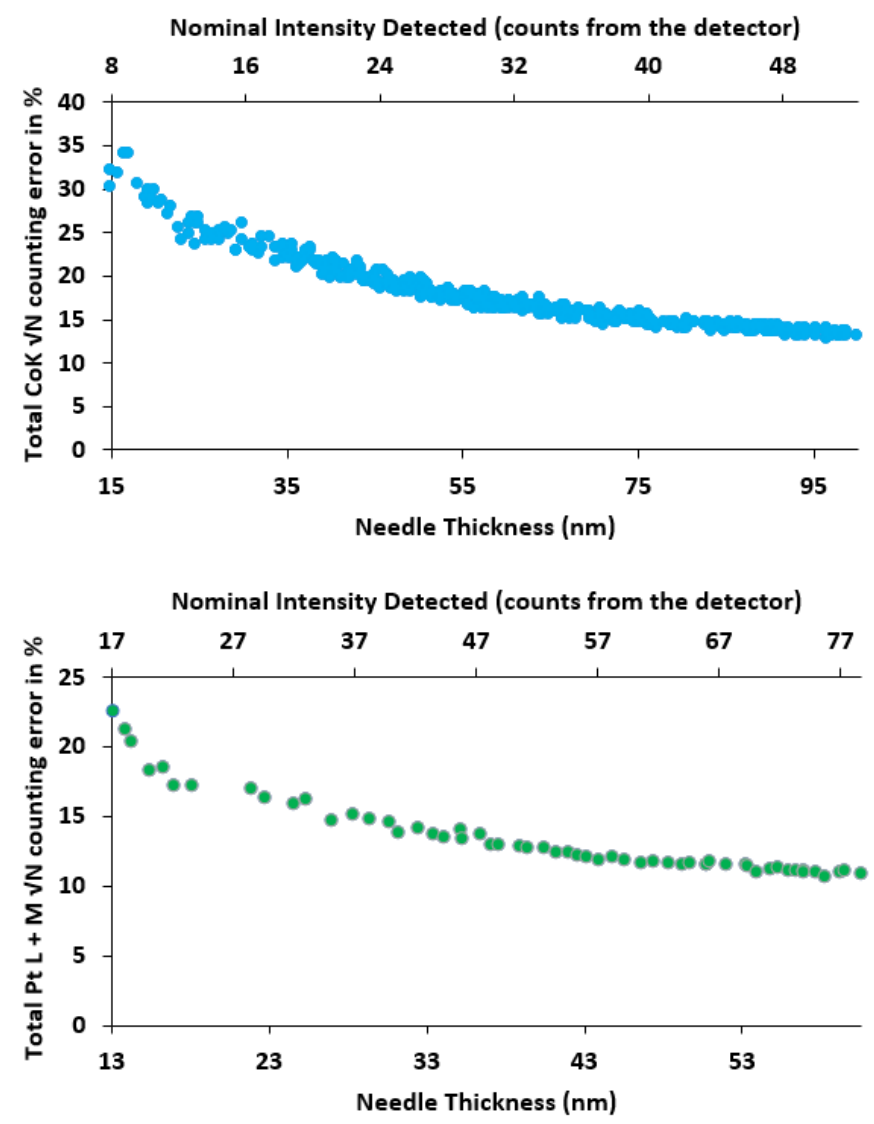
Figure 11 Percentage error from counting statistics for the needle samples as a function of thickness and nominal intensity.

\subsection{Error propagation for EDS several measurements}

There are several sources of error to consider for a single scattering partial cross-section measurement. To improve the statistical accuracy, several EDS measurements need to be taken. Alternatively, as shown by the previous section, the dose could also be increased. For several measurements the error in the partial cross-section determination $\sigma$ can be expressed as the standard error $S E$ for $n$ measurements with standard deviation $s$, equation 11 .

$$
\begin{gathered}
s^{2}=\sum_{j=1}^{n} \frac{\left(\sigma_{j}-\bar{\sigma}\right)}{n-1} \\
S E=\frac{s}{\sqrt{n}}
\end{gathered}
$$

The error measurements in Figure 9 and Figure 10, show that the individual error remains consistent after a certain sample thickness. This value depends on the dose of course and the largest contributor to this error is the counting statistics. However, since each error is similar, the above equation can be used to evaluate the total error in the process. If the error varies significantly with thickness for a given dose, then a weighted variance approach must be used (Galassi, 2009). This weighting was not implemented in this case as Figure 9 and Figure 10 show that the error remains consistent after a certain thickness value. Therefore, using equation 11, the average standard errors for the EDS partial cross-section was calculated from several measured line scans.

\subsection{EELS error propagation}

In the EELS partial cross-section calculation there are many possible sources of major error contributors. As the EELS signal is not dominated by counting statistics errors at the given 'nanoparticle conditions' dose here, it is not possible to provide the same treatment as the EDS error propagation. The major sources of error in EELS are systematic and the upper bounds of these can be quantified. Thickness, number density and magnification error contributions can be treated in the same manner as the EDS propagation show previously.

Error contributions from the chromatic aberration and collection angle determination are also negligible if the subsequent composition analysis is performed under the same microscope conditions. This error is effectively minimised by optimising the post specimen optics using a method proposed by (Craven et al., 2017) and directly measuring the collection angle proposed by (Jones et al., 2017).

The error that is difficult to quantify is the CCD gain and dark reference, for this a more detailed study is required. Here independent dark and gain references should be taken and compared to the automated Gatan routines. The error from the gain reference should cancel out in the subsequent fractionation and is expected to be negligible. The error arising from the dark reference correction is much more difficult to quantify as this would depend on several acquisition parameter such as the total SI time, and experiment mode used. This will be explored in a future study using the methods proposed by (Jones et al., 2016).

Surface oxidation and contamination could also potentially contribute to the error, however, these can be effectively minimised during the data processing stage (Craven et al., 2016). The largest source of error in the EELS partial cross-section arises from modelling the EELS background and its subtraction from the SI. In this case an inverse power law background was fit before the element edge. 
The error arising from this could vary between $1 \%-10 \%$ or greater depending on the signal to noise ratio and the edge/onset windows chosen (Liu and Brown, 1987). This is difficult to generalise if one wants to quantify samples such as nanoparticles, where the signal is very low.

A straightforward approach to calculate the overall error for the EELS partial cross-section could be calculated by checking the linearity of the data and its residuals with respect to the line of best fit and its confidence intervals Figure 12. From the standard error of the residuals gives an error of $0.002 \%$ for Co and $0.001 \%$ for Pt. This error is mostly negligible and therefore the main contribution to the partial cross-section measurement arises from the thickness, number density and magnification.

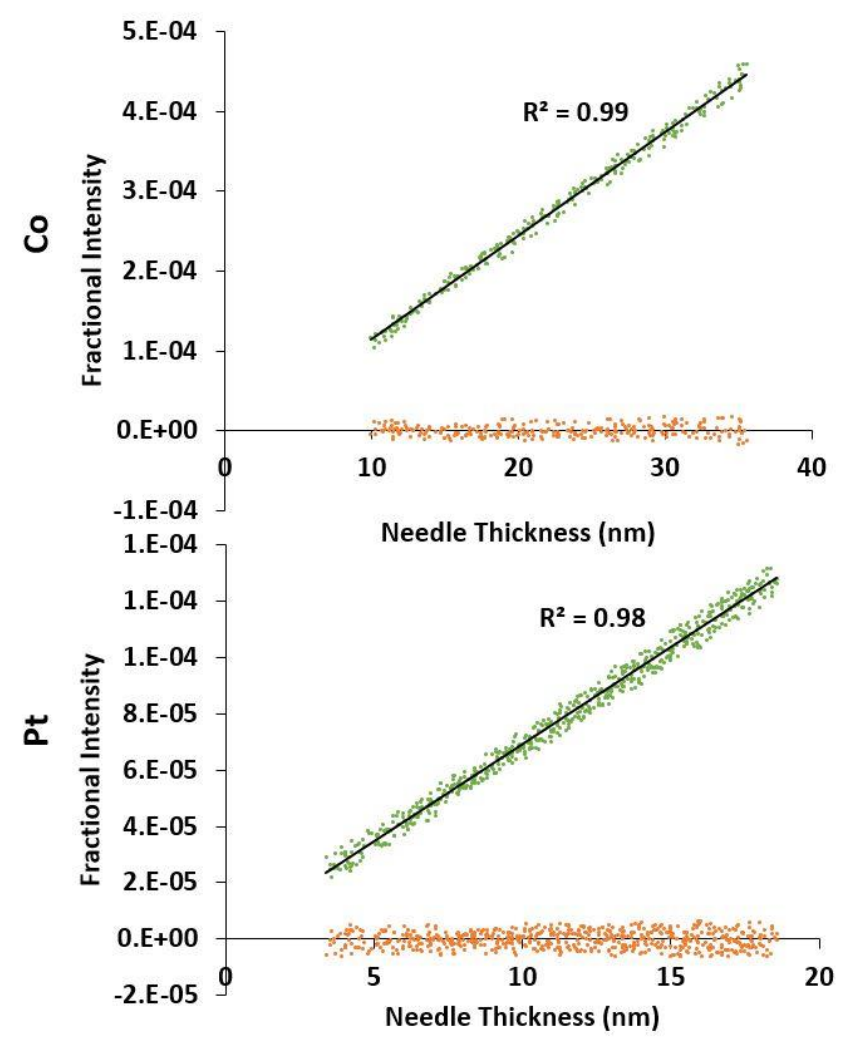

Figure 12 EELS fractional intensity plots from Figure 5 with their residuals and r-squared values. The green data points show the fractional intensity as a function of thickness, and the orange data points show the corresponding residuals.

\subsection{Percentage error summary}

The standard errors for the EDS and EELS partial cross-sections are tabulated in percentage, Table 6. The tabulated $\%$ errors give a good illustration of how the partial cross-section measurements vary between different detectors, sample types, and different beam currents given in Table 2. For instance, in the single EDS detector system, the relative Pt $L \alpha$ and $L \beta$ errors for the needle samples are lower than the nanoparticles due to several measurements adding to the confidence limit of the line fit in Figure 5.

None-the-less the EDS nanoparticle \% errors show good agreement compared to the needle samples. The nanoparticle \% errors are derived from an average of counting statistics, whereas the needle errors were obtained from a standard error of linescans. However, due to absorption effects the error in characterising the Pt $\mathrm{M} \alpha+\mathrm{M} \beta$ increases for the needle. In comparison the nanoparticle Pt $\mathrm{M} \alpha+\mathrm{M} \beta$ errors for the nanoparticles are much lower.

The largest contributor to the EDS error originates from the electron fluence which affects the signal to noise ratio. For the double EDS detectors, a current of $\sim 67 \mathrm{pA}$ was used resulting in a higher 
relative error due to counting statistics, whereas the single EDS detector measurements had a probe current of $\sim 186 \mathrm{pA}$. This once again highlights the importance of balancing total sample dose against sample damage to obtain reliable quantitative measurements.

For the EELS errors the largest contributors are systematic, this includes: the determination of sample thickness, number density and magnification. The upper bounds of these errors are quoted in the table. There can also be an additional error contribution (up to 10\%) from the modelling of the background and this error is edge dependent. Higher energy edges would be affected more compared to lower energy edges. Additionally, the signal to noise ratio would also contribute to the background model, especially for nanoparticles where the sample thickness and dose used is lower.

\begin{tabular}{|c|c|c|c|c|c|}
\hline \multirow{10}{*}{ 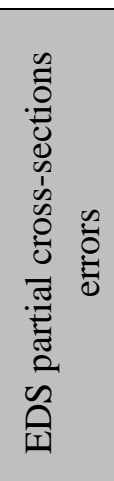 } & \multicolumn{5}{|c|}{ Pt EDS relative \% error with a single EDS detector } \\
\hline & EDS lines & $\mathrm{L} \alpha \quad \mathrm{L} \beta$ & $\mathrm{L} \alpha+\mathrm{L} \beta$ & $\mathrm{M} \alpha+\mathrm{M} \beta$ & $\mathrm{L}+\mathrm{M}$ \\
\hline & Needle & $3 \%$ & $3.4 \%$ & $8 \%$ & $6 \%$ \\
\hline & Nanoparticles & $6 \%$ & $5 \%$ & $3 \%$ & $3 \%$ \\
\hline & \multicolumn{5}{|c|}{ Pt EDS relative \% error with double EDS detectors } \\
\hline & \multirow{2}{*}{$\begin{array}{l}\text { EDS lines } \\
\text { Nanoparticles } \\
\end{array}$} & $\mathrm{L} \beta$ & $\mathrm{L} \alpha+\mathrm{L} \beta$ & $\mathrm{M} \alpha+\mathrm{M} \beta$ & $\mathrm{L}+\mathrm{M}$ \\
\hline & & $19 \%$ & $8 \%$ & $9 \%$ & $8 \%$ \\
\hline & \multicolumn{5}{|c|}{ Co EDS relative \% error with a single EDS detector } \\
\hline & \multirow{2}{*}{$\begin{array}{l}\text { EDS lines } \\
\text { Needle }\end{array}$} & $\mathrm{K} \beta$ & $K \alpha+K \beta$ & & \\
\hline & & $12 \%$ & $12 \%$ & & \\
\hline \multirow{7}{*}{ 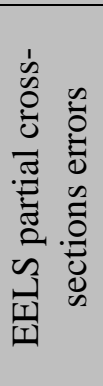 } & \multicolumn{5}{|c|}{ Pt EELS relative \% error } \\
\hline & \multirow{3}{*}{$\begin{array}{l}\text { EELS edges } \\
\text { Needle }\end{array}$} & \multicolumn{4}{|c|}{ M5, M4 (300eV window) } \\
\hline & & \multirow[t]{2}{*}{ Measured: $4 \%$} & \multirow{2}{*}{\multicolumn{2}{|c|}{$\begin{array}{l}\text { Calculated Hartree Slater: } \\
\text { Calculated Hydrogenic: }\end{array}$}} & --- \\
\hline & & & & & --- \\
\hline & \multicolumn{5}{|c|}{ Co EELs relative \% error } \\
\hline & \multirow{2}{*}{$\begin{array}{l}\text { EELS edges } \\
\text { Needle }\end{array}$} & \multicolumn{4}{|c|}{ L3, L2 (30eV window) } \\
\hline & & Measured: $3.5 \%$ & $\begin{array}{r}\text { Calculated } \\
\text { Calculat }\end{array}$ & $\begin{array}{l}\text { artree Slater: } \\
\text { Hydrogenic: }\end{array}$ & $\begin{array}{l}--- \\
---\end{array}$ \\
\hline
\end{tabular}

Table 6, The \% standard errors in the EDS and EELS partial cross-sections

\section{Conclusions}

A method of measuring and combining EDS and EELS signals using standard needle and nanoparticle standards is presented. The approach describes the intensity measured by the detector in terms of a partial scattering cross-section. These cross-sections are partial cross-sections that depend on several microscope parameters such as spectrum integration-window, detector efficiency, collection-angle and size. By representing the scattering cross-sections in 'barns' allows for an easier benchmarking between different microscopes, which is otherwise not fully realised using the solid angle benchmark.

The spectroscopic partial cross-sections from the needle and nanoparticle standards agree well and both methods could be used as calibration standards provided that the number density of the samples is determined accurately. Error analysis of the individual partial cross-section measurements shows that counting statistics could lead to an error of up to $20 \%$ when determining absolute number of atoms. This can be reduced by increasing the dose, however sample damage would also increase. A balance between dose and sample damage is required and would by useful to investigate in another study. This means that for quantifying on axis nanoparticles in the future with the current hardware the error per column count can range from 2 atoms up to 5 atoms.

When comparing the measured EELS partial cross-sections to the HS cross-sections the agreement between the Hartree-Slater cross-sections and the measured EELS cross-sections is within 20-40\% depending on the integration windows used and the edge shape (white-lines). It is reassuring that the EELS cross-section calculations using Digital Micrograph provide reasonable values that can be used 
for quantification. The reliability of the measured EDS partial cross-sections was verified with an independent ADF quantification. In this comparison, a pure on-axis Pt nanoparticle was atom-counted using both EDS and ADF quantification and the total number of atoms agreed well. The precision in using EDS to count the number of atoms in an individual column was very poor however due to counting statistics. The resolution and SNR can be improved further using multiframe acquisition. In the future this will most likely be the routine approach to quantify spectroscopic signals.

By unifying the ADF, EDS and EELS signals in terms of scattering partial cross-section it is possible to directly compare and switch between the different acquisition modes seamlessly. This method will pave the way for measuring number of atoms for catalyst nanoparticles by exploiting detector efficiencies to atom count bimetallic nanoparticles with high throughput in a future study.

\section{Acknowledgements}

The authors would like to thank the EPSRC and Johnson Matthey for funding this work as part of a CASE-Award studentship. The research leading to these results has received funding from the European Union Seventh Framework Programme under Grant Agreement 312483 - ESTEEM2 (Integrated Infrastructure Initiative-I3). We would like to thank Brian Theobald and Jonathan Sharman from JMTC for provision of the Pt nanoparticle samples. We wold also like to thank Manfred Schuster and Dogan Ozkaya for access to the double EDS ARM 200CF. We would also like to thank Hidetaka Sawada from JEOL for helping us with the projector lens optimisation experiment. Some of the experimental work was performed on the South of England Analytical Electron Microscope funded through EPSRC grant EP/K040375/1.

\section{Competing Interests}

The authors declare that they have no competing interests.

\section{References}

Aarons, J., Jones, L., Varambhia, A., MacArthur, K.E., Ozkaya, D., Sarwar, M., Skylaris, C.K., Nellist, P.D., 2017. Predicting the Oxygen-Binding Properties of Platinum Nanoparticle Ensembles by Combining High-Precision Electron Microscopy and Density Functional Theory. Nano Lett. 17, 4003-4012. doi:10.1021/acs.nanolett.6b04799

Allen, L.J., D’Alfonso, A.J., Freitag, B., Klenov, D.O., 2012. Chemical mapping at atomic resolution using energy-dispersive x-ray spectroscopy. MRS Bull. 37, 47-52. doi:10.1557/mrs.2011.331

Allen, L.J., D'Alfonso, A.J., Findlay, S.D., 2014. Modelling the inelastic scattering of fast electrons. Ultramicroscopy 151, 1-12. doi:10.1016/j.ultramic.2014.10.011

Bobynko, J., MacLaren, I., Craven, A.J., 2015. Spectrum imaging of complex nanostructures using DualEELS: I. digital extraction replicas. Ultramicroscopy 149, 9-20. doi:10.1016/j.ultramic.2014.10.014

Chen, Z., Weyland, M., Sang, X., Xu, W., Dycus, J.H., LeBeau, J.M., D’Alfonso, A.J., Allen, L.J., Findlay, S.D., 2016. Quantitative atomic resolution elemental mapping via absolute-scale energy dispersive X-ray spectroscopy. Ultramicroscopy 168, 7-16. doi:10.1016/j.ultramic.2016.05.008

Cliff, G., Lorimer, G.W., 1975. The quantitative analysis of thin specimens. J. Microsc. 103, 203-207. doi:10.1111/j.1365-2818.1975.tb03895.x

Craven, A.J., Bobynko, J., Sala, B., MacLaren, I., 2016. Accurate measurement of absolute experimental inelastic mean free paths and EELS differential cross-sections. Ultramicroscopy 170, 113-127. doi:10.1016/j.ultramic.2016.08.012

Craven, A.J., Sawada, H., McFadzean, S., MacLaren, I., 2017. Getting the most out of a post-column EELS spectrometer on a TEM/STEM by optimising the optical coupling. Ultramicroscopy 180 , 
66-80. doi:10.1016/j.ultramic.2017.03.017

Davey, W.P., 1925. Precision Measurements of the Lattice Constants of Twelve Common Metals. Phys. Rev. 25, 753-761. doi:10.1103/PhysRev.25.753

De Backer, A., Martinez, G.T., Rosenauer, A., Van Aert, S., 2013. Atom counting in HAADF STEM using a statistical model-based approach: Methodology, possibilities, and inherent limitations. Ultramicroscopy 134, 23-33. doi:10.1016/j.ultramic.2013.05.003

De Backer, A., van den Bos, K.H.W., Van den Broek, W., Sijbers, J., Van Aert, S., 2016. StatSTEM: An efficient approach for accurate and precise model-based quantification of atomic resolution electron microscopy images. Ultramicroscopy 171, 104-116. doi:10.1016/J.ULTRAMIC.2016.08.018

De wael, A., De Backer, A., Jones, L., Nellist, P.D., Van Aert, S., 2017. Hybrid statistics-simulations based method for atom-counting from ADF STEM images. Ultramicroscopy 177, 69-77. doi:10.1016/j.ultramic.2017.01.010

E, H., MacArthur, K.E., Pennycook, T.J., Okunishi, E., D’Alfonso, a. J., Lugg, N.R., Allen, L.J., Nellist, P.D., 2013. Probe integrated scattering cross sections in the analysis of atomic resolution HAADF STEM images. Ultramicroscopy 133, 109-119. doi:10.1016/j.ultramic.2013.07.002

Egerton, R.F., 2011. Electron Energy-Loss Spectroscopy in the Electron Microscope, Springer, New York. doi:10.1007/978-1-4419-9583-4

Egerton, R.F., 2008. Electron energy-loss spectroscopy in the TEM. Reports Prog. Phys. 72, 016502. doi:10.1088/0034-4885/72/1/016502

Findlay, S.D., Chen, Z., Weyland, M., Sang, X., Xu, W., Dycus, J.H., LeBeau, J.M., Allen, L.J., 2017. Absolute-Scale Comparison with Simulation for Quantitative Energy-Dispersive X-Ray Spectroscopy in Atomic-Resolution Scanning Transmission Electron Microscopy. Microsc. Microanal. 23, 388-389. doi:10.1017/S1431927617002628

Galassi, M., 2009. GNU scientific library : reference manual. Network Theory.

Gubbens, A., Barfels, M., Trevor, C., Twesten, R., Mooney, P., Thomas, P., Menon, N., Kraus, B., Mao, C., McGinn, B., 2010. The GIF Quantum, a next generation post-column imaging energy filter. Ultramicroscopy 110, 962-970. doi:10.1016/j.ultramic.2010.01.009

Hofer, F., 1991. Determination of inner-shell cross-sections for EELS-quantification. Microsc. Microanal. Microstruct. 2, 215-230. doi:10.1051/mmm:0199100202-3021500

hyperspy/hyperspy: HyperSpy 1.3, 2017. doi:10.5281/ZENODO.583693

Iakoubovskii, K., Mitsuishi, K., Nakayama, Y., Furuya, K., 2008. Thickness measurements with electron energy loss spectroscopy. Microsc. Res. Tech. 71, 626-631. doi:10.1002/jemt.20597

Jones, L., MacArthur, K.E., Fauske, V.T., van Helvoort, A.T.J., Nellist, P.D., 2014. Rapid estimation of catalyst nanoparticle morphology and atomic-coordination by high-resolution z-contrast electron microscopy. Nano Lett. 14, 6336-41. doi:10.1021/nl502762m

Jones, L., Varambhia, A., Beanland, R., Kepaptsoglou, D., Griffiths, I., Ishizuka, A., Azough, F., Freer, R., Ishizuka, K., Cherns, D., Ramasse, Q.M., Lozano-Perez, S., Nellist, P.D., 2018. Managing dose-, damage- and data-rates in multi-frame spectrum-imaging. Microscopy. doi:10.1093/JMICRO/DFX125

Jones, L., Varambhia, A., Kepaptsoglou, D., Ramasse, Q., Freer, R., Azough, F., Lozano-Perez, S., Beanland, R., Nellist, P., 2016. New Opportunities in multi-frame STEM Spectroscopy \&amp; Fractional Beam-current EELS, in: European Microscopy Congress 2016: Proceedings. WileyVCH Verlag GmbH \& Co. KGaA, Weinheim, Germany, pp. 809-810. doi:10.1002/9783527808465.EMC2016.6336 
Jones, L., Varambhia, A., Sawada, H., Nellist, P.D., 2017. An optical configuration for fastidious STEM detector calibration and the effect of the objective lens pre-field Manuscript. J. Microsc. submitted.

Jones, L., Yang, H., Pennycook, T.J., Marshall, M.S.J., Van Aert, S., Browning, N.D., Castell, M.R., Nellist, P.D., 2015. Smart Align - a new tool for robust non-rigid registration of scanning microscope data. Adv. Struct. Chem. Imaging 1, 8. doi:10.1186/s40679-015-0008-4

Koh, S., Strasser, P., 2007. Electrocatalysis on bimetallic surfaces: Modifying catalytic reactivity for oxygen reduction by voltammetric surface dealloying. J. Am. Chem. Soc. 129, 12624-12625. doi:10.1021/ja0742784

Kothleitner, G., Grogger, W., Dienstleder, M., Hofer, F., 2014. Linking TEM Analytical Spectroscopies for an Assumptionless Compositional Analysis. Microsc. Microanal. 20, 678-86. doi:10.1017/S1431927614000130

Kothleitner, G., Hofer, F., 1998. Optimization of the Signal to Noise Ratio in EFTEM Elemental Maps with Regard to Different Ionization Edge Types. Micron 29, 349-357. doi:10.1016/S09684328(98)00014-6

Lábár, J.L., Salter, C.J., 1991. Uncertainties in the Analysis of M X-Ray Lines of the Rare-Earth Elements, in: Electron Probe Quantitation. Springer US, Boston, MA, pp. 223-249. doi:10.1007/978-1-4899-2617-3_13

Leapman, R., Rez, P., Mayers, D., 1980. K, L, and M shell generalized oscillator strengths and ionization cross sections for fast electron collisions. J. Chem. Phys. 72, 1232. doi:10.1063/1.439184

Liu, D.-R., Brown, L.M., 1987. Influence of some practical factors on background extrapolation in EELS quantification. J. Microsc. 147, 37-49. doi:10.1111/j.1365-2818.1987.tb02816.x

Liu, J., Cowley, J.M., 1990. High-angle ADF and high-resolution SE imaging of supported catalyst clusters. Ultramicroscopy 34, 119-128. doi:10.1016/0304-3991(90)90066-U

Liu, Z., Ma, L., Zhang, J., Hongsirikarn, K., Goodwin, J.G., 2013. Pt Alloy Electrocatalysts for Proton Exchange Membrane Fuel Cells: A Review. Catal. Rev. 55, 255-288. doi:10.1080/01614940.2013.795455

MacArthur, K.E., Brown, H.G., Findlay, S.D., Allen, L.J., 2017. Probing the effect of electron channelling on atomic resolution energy dispersive X-ray quantification. Ultramicroscopy 182, 264-275. doi:10.1016/J.ULTRAMIC.2017.07.020

Macarthur, K.E., Slater, T.J.A., Haigh, S.J., Ozkaya, D., Nellist, P.D., Lozano-perez, S., 2015. Quantitative EDX Analysis of Catalyst Nanoparticles Using a Partial Scattering Cross Section Approach. Microsc. Microanal. In press, 1-23. doi:10.1017/S1431927615015494

Malis, T., Cheng, S.C., Egerton, R.F., 1988. EELS log-ratio technique for specimen-thickness measurement in the TEM. J. Electron Microsc. Tech. 8, 193-200. doi:10.1002/jemt.1060080206

Martinez, G.T., Jones, L., Backer, A. De, Béché, A., Verbeeck, J., Aert, S. Van, Nellist, P.D., 2015. Quantitative STEM normalisation: the importance of the electron flux. Ultramicroscopy. doi:10.1016/j.ultramic.2015.07.010

Miller, M.K., Russell, K.F., 2007. Atom probe specimen preparation with a dual beam SEM/FIB miller. Ultramicroscopy 107, 761-6. doi:10.1016/j.ultramic.2007.02.023

Nellist, P.D., 2011. Scanning Transmission Electron Microscopy: Imaging and Analysis. Chapter 2, Springer. doi:10.100/978-1-4419-7-200-2

Nord, M., Vullum, P.E., MacLaren, I., Tybell, T., Holmestad, R., 2017. Atomap: a new software tool for the automated analysis of atomic resolution images using two-dimensional Gaussian fitting. 
Adv. Struct. Chem. Imaging 3, 9. doi:10.1186/s40679-017-0042-5

Ostaševičius, T., de la Peña, F., Midgley, P., 2016. SAMFire - a smart adaptive fitting algorithm for multi-dimensional microscopy, in: European Microscopy Congress 2016: Proceedings. WileyVCH Verlag GmbH \& Co. KGaA, Weinheim, Germany, pp. 95-96. doi:10.1002/9783527808465.EMC2016.6233

Owen, E.A., Jones, D.M., 1954. Effect of Grain Size on the Crystal Structure of Cobalt. Proc. Phys. Soc. Sect. B 67, 456-466. doi:10.1088/0370-1301/67/6/302

Phillips, P.J., Paulauskas, T., Rowlands, N., Nicholls, A.W., Low, K.-B., Bhadare, S., Klie, R.F., 2014. A New Silicon Drift Detector for High Spatial Resolution STEM-XEDS: Performance and Applications. Microsc. Microanal. 20, 1046-52. doi:10.1017/S1431927614001639

Retsky, M., 1974. Observed single atom elastic cross sections in a scanning electron microscope. Optik (Stuttg). 41, 127-142. doi:10.2172/4239746

Rosenauer, A., Gries, K., Müller, K., Pretorius, A., Schowalter, M., Avramescu, A., Engl, K., Lutgen, S., 2009. Measurement of specimen thickness and composition in Alx Ga1 - x N / GaN using high-angle annular dark field images. Ultramicroscopy 109, 1171-1182. doi:10.1016/j.ultramic.2009.05.003

Rosenauer, A., Mehrtens, T., Müller, K., Gries, K., Schowalter, M., Satyam, P.V., Bley, S., Tessarek, C., Hommel, D., Sebald, K., Seyfried, M., Gutowski, J., Avramescu, A., Engl, K., Lutgen, S., 2011. Composition mapping in InGaN by scanning transmission electron microscopy. Ultramicroscopy 111, 1316-27. doi:10.1016/j.ultramic.2011.04.009

Tait, E.W., Ratcliff, L.E., Payne, M.C., Haynes, P.D., Hine, N.D.M., 2016. Simulation of electron energy loss spectra of nanomaterials with linear-scaling density functional theory. J. Phys. Condens. Matter 28, 195202. doi:10.1088/0953-8984/28/19/195202

Thompson, W.J., 2001. Poisson distributions. Comput. Sci. Eng. 3, 78-82. doi:10.1109/5992.919271

Van Aert, S., Batenburg, K.J., Rossell, M.D., Erni, R., Van Tendeloo, G., 2011. Three-dimensional atomic imaging of crystalline nanoparticles. Nature 470, 374-377. doi:10.1038/nature09741

Watanabe, M., Horita, Z., Nemoto, M., 1996. Absorption correction and thickness determination using the zeta factor in quantitative X-ray microanalysis. Ultramicroscopy 65, 187-198. doi:10.1016/S0304-3991(96)00070-8

Watanabe, M., Williams, D.B., 2006. The quantitative analysis of thin specimens: A review of progress from the Cliff-Lorimer to the new $\zeta$-factor methods. J. Microsc. 221, 89-109. doi:10.1111/j.1365-2818.2006.01549.x

Watanabe, M., Williams, D.B., Tomokiyo, Y., 2003. Comparison of detectability limits for elemental mapping by EF-TEM and STEM-XEDS. Micron 34, 173-183. doi:10.1016/S09684328(03)00028-3

Xin, H.L., Mundy, J.A., Liu, Z., Cabezas, R., Hovden, R., Kourkoutis, L.F., Zhang, J., Subramanian, N.P., Makharia, R., Wagner, F.T., Muller, D.A., 2012. Atomic-resolution spectroscopic imaging of ensembles of nanocatalyst particles across the life of a fuel cell. Nano Lett. 12, 490-497. doi:10.1021/nl203975u

Zanaga, D., Altantzis, T., Sanctorum, J., Freitag, B., Bals, S., 2016. An alternative approach for $\zeta$ factor measurement using pure element nanoparticles. Ultramicroscopy 164, 11-16. doi:10.1016/j.ultramic.2016.03.002

Zhang, H.-R., Egerton, R.F., Malac, M., 2012. Local thickness measurement through scattering contrast and electron energy-loss spectroscopy. Micron 43, 8-15.

doi:10.1016/j.micron.2011.07.003 
